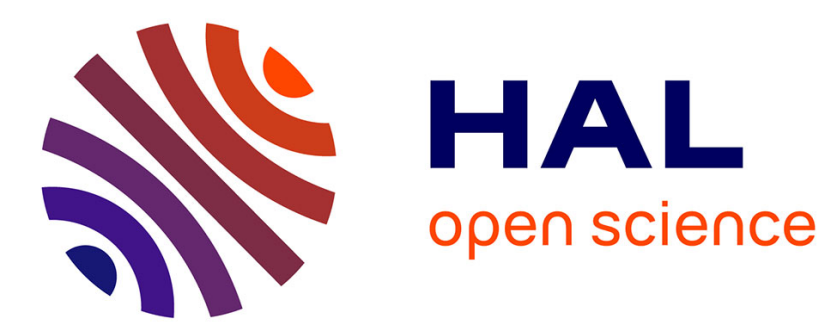

\title{
Policy Measures and Effects in the Different Phases of the Cluster Life-Cycle
}

\author{
Thomas Brenner, Charlotte Schlump
}

\section{To cite this version:}

Thomas Brenner, Charlotte Schlump. Policy Measures and Effects in the Different Phases of the Cluster Life-Cycle. Regional Studies, 2011, pp.1. 10.1080/00343404.2010.529116 . hal-00658168

\section{HAL Id: hal-00658168 \\ https://hal.science/hal-00658168}

Submitted on 10 Jan 2012

HAL is a multi-disciplinary open access archive for the deposit and dissemination of scientific research documents, whether they are published or not. The documents may come from teaching and research institutions in France or abroad, or from public or private research centers.
L'archive ouverte pluridisciplinaire HAL, est destinée au dépôt et à la diffusion de documents scientifiques de niveau recherche, publiés ou non, émanant des établissements d'enseignement et de recherche français ou étrangers, des laboratoires publics ou privés. 


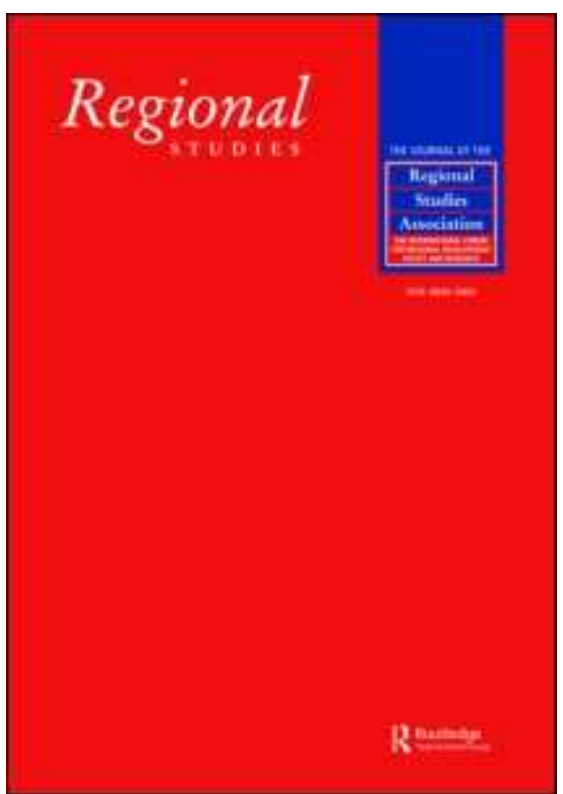
Policy Measures and Effects in the Different Phases of the
Cluster Life-Cycle

\begin{tabular}{|r|l|}
\hline Journal: & Regional Studies \\
\hline Manuscript ID: & CRES-2009-0137.R1 \\
\hline Manuscript Type: & Main Section \\
\hline JEL codes: & $\begin{array}{l}\text { R12 - Size and Spatial Distributions of Regional Economic Activity }< \\
\text { R1 - General Regional Economics }<\text { R - Urban, Rural, and Regional } \\
\text { Economics, C51 - Model Construction and Estimation }<\text { C5 - } \\
\text { Methods, C53 - Forecasting and Other Model Applications }<\text { C5 }- \\
\text { Econometric Modeling }<\text { C - Mathematical and Quantitative Methods }\end{array}$ \\
\hline \hline Keywords: & cluster, life-cycle, mathematical model \\
\hline
\end{tabular}

\section{SCHOLARONE" Manuscripts}


Policy Measures and Their Effects in the Different Phases of the Cluster Life-Cycle Thomas Brenner and Charlotte Schlump

Current address:

Philipps-University Marburg, Department of Geography, Deutschhausstr. 10, 35032 Marburg, Germany

thomas.brenner@staff.uni-marburg.de, tel. +49-6421-2824211, fax.+49-6421-2828950 schlump@staff.uni-marburg.de, tel. +49-6421-2824409, fax. +49-6421-2828950

(Received May 2009: in revised form June 2010) 


\begin{abstract}
In recent years, policy measures have frequently been applied to clusters. This paper analyses whether different types policy measures should be applied in different stages of the cluster life-cycle. Two approaches are used to obtain answers to this question. First, we gather insights from existing literature on policy measures and their evaluation. Second, we set up a mathematical model in order to examine the effects of various policy measures in the different life-cycle phases. We find that the adequate kind of policy measures depend on the cluster's current stage in its life-cycle.
\end{abstract}

Keywords: cluster, life-cycle, policy

JEL: C50, O30, R11, R58

\begin{abstract}
German:
Die Förderung von Clustern hat in den letzten Jahren in der Politik viel Aufmerksamkeit erhalten. Dieser Artikel untersucht mit zwei verschiedenen Ansätzen, ob in verschiedenen Phasen des Clusterlebenszyklus unterschiedliche Fördermaßnahmen geeignet sind. Zunächst werden die in der Literatur vorhandenen Ergebnisse und Erkenntnisse über Fördermaßnahmen und deren Wirksamkeit zusammengetragen. In einem zweiten Schritt wird ein mathematisches Modell der Clusterentwicklung aufgestellt. Mit Hilfe dieses Modells werden die Effekte der verschiedenen Fördermaßnahmen in den unterschiedlichen Phasen des Clusterlebenszyklus analysiert. Dabei können wir zeigen, dass eine adäquate Förderung von Clustern tatsächlich von der aktuellen Phase im Lebenszyklus abhängt.
\end{abstract}




\section{Introduction}

Currently, there has been a growing interest in generating and supporting local clusters. While designing policy programs, policy makers often do not take into account the different phases of the cluster life-cycle. The same measures are applied to all supported local clusters within a program. Hence, if the choice of clusters within such programs does not implicitly restrict the applied measure to clusters in the same life-cycle phase, no differentiation between cluster life-cycle phases is made.

This is partly caused by the fact that the scientific literature rarely provides recommendations on how to select adequate policy measures in order to support clusters throughout the different stages of the cluster life-cycle. While there does exist sufficient literature that which deals with the cluster life-cycle as such, the connection between lifecycle theory and policy has been mainly ignored. This paper intends to fill part of this gap. There is an ongoing debate about the effectiveness and usefulness of cluster policy. While some authors assume governmental support only to have small influence on clusters' emerge or competitiveness (e.g. VAN DER LINDE, 2003), others tend to see great potential in governmental intervention. As most of the policy activities on clusters have been conducted quite recent, the measures' impact still needs time to show up and to be evaluated (ENRIGHT, 2003). Furthermore, we expect that different policy measures vary in their effectiveness over the cluster life-cycle since mechanisms and processes relevant within clusters change over time. Economically successful and competitive clusters usually do not need any policy support, whereas certain measures might be conducive to the functioning of a cluster during its emergence and maturity phase in particular (HOSPERS and BEUGELSDIJK, 2002).Thus, it is not easy to determine what kind of policy measures really affect cluster development as the needs and evolution of clusters vary between the different types and stages of the cluster's life-cycle.

Different policy measures are examined in varying depths in the literature. Universities and 
research institutes are seen as major driving forces of cluster formation. Hence, their relevance in different stages of the cluster development is frequently discussed (HUGGINS, 2008; MARTIN and SUNLEY, 2003 among others). Other aspects of policy involvement, such as the support of local cooperation, are rarely addressed in the context of the cluster life-cycle. Furthermore, the discussion is entirely theoretical. There is a lack of empirical comparisons and mathematical approaches evaluating the different effects of policy measures in the stages of the cluster life-cycle.

Our approach differs from the existing literature in various aspects. First, we develop a mathematical model that describes the dynamics of clustering. We use this model to analyse the impact of policy measures in the different stages of the cluster life-cycle. Second, we use empirical data to set up and calibrate the model, i.e. the mechanisms causing cluster formation are modelled in line with empirical knowledge in order to make the implications of the model reliable. We also estimate the parameters of the model empirically in order to make the magnitude of effects reliable. Third, we use knowledge from the literature to determine the changes of the model's parameters over the cluster life-cycle. This leads to reliable implications on the effectiveness of policy along the cluster life-cycle.

This paper aims at a better understanding of the impact of policy measures on the development of local clusters in general and of the relevance of the measures' timing in particular. The key contribution of this paper to the literature on cluster life-cycles is the answer to the question of how policy measures should vary along the different stages of the cluster life-cycle. The results allow us to advice policy makers on adequate policy measures. We believe that policy activities should take into account the different stage of the cluster life-cycle.

The paper proceeds as follows. In Section II we reflect on the theory of the cluster lifecycle and the existing empirical and theoretical knowledge about policy measures and 
their impact. The mathematical model is set up in Section III. In Section IV the general characteristics of this model are analysed first. Successively, the impacts of the various policy measures are examined. Section $\mathrm{V}$ concludes the paper.

\section{Theory}

There is a huge literature on clusters and a small but developing literature on the cluster life-cycle. This literature describes the development of clusters in time. Various theories and concepts on cluster development exist. They build their arguments on different ideas, theories, and empirical observations. Nevertheless, there is quite an overlap in the predictions. In the following, we discuss the basic arguments and predictions about the cluster life-cycle and detect a number of common features.

Furthermore, different policy measures that are applied to clusters are analyzed and grouped. In this context we focus on our main question discussing the effectiveness of different policy measures along the cluster life-cycle. There is an ongoing debate about the effectiveness of cluster policy in general. A quantitative survey of all possible cluster policies and a comparison of their impacts are still missing (KIESE, 2008). Quite often, policy or public action is seen as an inevitable and internal element of clusters (BORRÁS and TSAGDIS, 2008). This shows the implicitness of some policy intervention and the difficulty to estimate the overall importance of policy for the enhancement of cluster capabilities and competitiveness. Considering all knowledge about clusters and policy involvement, especially knowledge gathered in case studies (see BRENNER and MÜHLIG, 2007) we come to the conclusion that policy measures at least have a potential impact on the development of clusters. In this paper, we do not focus on the question of whether there are policy impacts, but on the question of how the timing and choice of measures can help to make these more effective. 
Therefore, we start with a description of the cluster life-cycle (Section II.1). Then we reflect on the discussion about the effectiveness of cluster policy (Section II.2). Although we do not intend to and cannot answer the question of whether cluster policy is effective, this discussion shows the importance of further analyses about the choice of the adequate measures. The various potential measures are discussed in detail in Section II.3.

\section{II.1 The cluster life-cycle}

The various approaches on the cluster life-cycle more or less seem to agree about the existence of three distinct phases: an initial or development stage, an expansion stage, in which the cluster forces that are usually discussed in the literature are active, and a mature stage. The last phase sometimes is combined with the option of decline (MAGGIONI, 2004). Further, the phase of decline sometimes is defined as a fourth stage, which might also be characterised by a transformation or a decline of the cluster (VAN KLINK and DE LANGEN, 2001 and BRENNER, 2001). Although there is a fourth stage, we only include the first three stages in our analysis since the mechanisms and processes underlying the fourth stage differ crucially, which would complicate the analysis of the model.

The cluster life-cycle concept has developed out of the concepts of the product and industry life-cycle. Nevertheless, cluster and industry or firm life-cycles are not the same. They have developed differently over time and only show some parallels (MENZEL and FORNAHL, 2007). However, as the industry life-cycle is connected with agglomeration effects and thus the formation of clusters, there is a linkage between the two concepts (AUDRETSCH and FELDMAN, 1996). BRENNER (2001 and 2008) argues that there is a causal relation between the industrial life-cycle and the life-cycle of clusters in this industry. Usually, clusters emerge during the initial stage of the corresponding industry life-cycle. A cluster's expansion phase usually goes together with the expansion phase of the market 
for the respective products. If an industry is mature and the markets satiated and very competitive, clusters usually stabilise and show little dynamics.

\section{Initial or development stage}

In the initial stage of the cluster life-cycle we are able to detect the emergence of some places in which the respective industry becomes concentrated. When a new industry or technology emerges, the first firms active in the emerging field are often scattered in space (KLEPPER, 2007). While the industry is growing, more firms appear and start to expand. There are certain forces that cause growth to be stronger in a few regions. Spin-offs can be seen as one of the mechanisms causing the geographic concentration of an industry (ARTHUR, 1994; KLEPPER, 2007). MENZEL and FORNAHL (2007) argue that in the initial stage only little interaction between firms in a region does take place; they see spinoffs and the interaction between firms and universities as well as firms and public research institutes as the main driving forces in this stage. Furthermore, local conditions and policy have an influence on the development of the cluster. On the contrary, BRUSCO (1990) argues that in the very beginning of the development of industrial districts government intervention is hardly feasible as in this stage it is impossible to identify clusters statistically and thus to apply appropriate policy measures. He argues that only later, when industrial districts are more established, there is a need for intervention and policy measures.

In the initial phase of the cluster life-cycle it is important that a critical mass of economic activity is reached in the region (ARTHUR, 1994; BRENNER, 2001). Local conditions as well as the kind and number of firms located in the region determine whether this critical mass is reached or not. Especially the founding of firms that become central for the region can be seen as a random event that contributes to the development of the cluster crucially (PORTER, 1998b). BOSCHMA and WENTING (2007) show that the presence of related industries also plays an important role for the further growth of clusters. 
To sum up, in the initial stage start-ups and spin-offs are of great importance but also the parent firm or organization may play an strong role. Furthermore, local conditions, especially the presence of related industries, universities, and public research seem to contribute to the development of clusters. This implies that all variables and parameters related to firm foundation, the presence of industries, universities and public research should be high in this early phase of the cluster life-cycle. According to BRENNER (2001) and MENZEL and FORNAHL (2007) the industry life-cycle is in its initial stage, too, so that the market situation can be expected to start improving.

\section{Expansion stage}

In the cluster's expansion stage, the market for the respective technology or industry increases tremendously and so do the number of firms, employment and other related activities. In this phase, Marshall's agglomeration economies become influential (MAGGIONI, 2004). Marshall argues that the local firm population benefits from a common labour market, synergies and interaction between firms and the development of service and supplier firms in the region. Part of this argument is based on spillovers between firms that seem to contribute to local growth, especially in 'new' industries (GLAESER et al. 1992, HENDERSON et al. 1995). These spillovers do not only occur between firms of the same industry, as argued by Marshall, but also between firms from different industries. The latter is part of the so-called Jacob's externalities. This implies that diversity becomes an important aspect in the development.

The networking activities grow and become very important in the expansion phase, while the cluster becomes more focused (MENZEL and FORNAHL, 2007). Furthermore, we know from the industrial life-cycle literature that innovation activities are very important in this phase (KLEPPER, 1997). This seems to apply for the cluster life-cycle as well. 
To sum up, in the expansion stage regional self-reinforcing processes become crucial for the developments in the region. These processes are mainly based on direct interaction between firms and their surroundings. Cooperation, networks, and innovations are very important in this phase. Start-ups are still important, although slightly loosing importance. Hence, all variables and parameters related to innovation and interaction between regional actors can be expected to have very high values, although those connected to start-up activities still have some importance. The market situation is very profitable in this phase.

\section{Mature stage}

In the mature stage of the cluster, market growth has slowed down (BRENNER, 2001). Market development has stabilised and the situation has reached a kind of equilibrium (MENZEL and FORNAHL, 2007). In the industry life-cycle, the system passes through a shake-out phase (KLEPPER, 1997). Start-ups do not play a role any more. We still find some regional self-reinforcing forces and firms might still benefit from being located in a cluster (as the finding in PORTER, 2003 suggest). However, there is also empirical evidence suggesting that firms do not benefit from being located in a cluster in later stages of the cluster life-cycle (AUDRETSCH and FELDMAN,1996). However, regional networking and cooperation activities are well established in this stage (MENZEL and FORNAHL, 2007).

To sum up, start-ups play no role in the mature stage of the cluster life-cycle anymore. Most cluster forces are only of some importance, while regional networking and cooperation activities are main features of well-established clusters. Hence, all variables and parameters connected with networking should be high, while most other variables and parameters are low or medium. 


\section{Declining stage}

Compared to the mature stage, we do not find much difference to the cluster's situation in the declining stage. This might be a reason why these two stages are sometimes not distinguished from each other. The main difference is that in the declining stage the market for the products produced within the cluster decreases strongly. Hence, the market situation becomes very difficult. Therefore, the cluster is forced to decline or to transform itself in order to open up new market possibilities.

It is difficult to study the declining stage with the model developed below. Therefore, we focus our analysis on the first three stages of the cluster life-cycle.

\section{Transition between stages}

In their development, clusters do not jump from one stage to the other. Instead, they usually transform slowly to the next stage. This transformation can be seen as a transition phases. As clusters consist of a huge amount of different firms and institutions, these actors are not necessarily in exactly the same stage of development at any point in time. Therefore, some protagonists may stay in a certain life-cycle stage whereas others already moved further on. We define the cluster life-cycle phase as the phase in which the focal firms are at each point in time (MENZEL and FORNAHL, 2007).

II.2 Cluster policy and policy considerations

Nowadays, nearly all national and regional authorities apply some kind of cluster policy measures. As clusters are generally said to provide advantages to regions and their firms, the overall aim of cluster policy is to enhance the economic performance of regions and nations (EUROPEAN COMMISSION, 2008; RAINES, 2000). Cluster policy can be seen as "a set of policy interventions aiming at strengthening existing clusters or facilitating the 
emergence of new ones" (EUROPEAN COMMISSION, 2008, p.28). There is an ongoing debate whether cluster policy really is a new policy field or just old wine in new skins. The seminal works of PORTER $(1990,1998 a, 1998 b)$ build the theoretical background for most of the measures promoting clusters in the recent years but there are also cluster-related policies with different background. Most of these ideas have been integrated in industrial policy in developed countries in the 1990s focusing on collaborative elements, networks, and regional developments. These cluster-related measures complement traditional sector policies by adding a spatial focus (BORRÁS and TSAGDIS, 2008; KOSCHATZKY, 2005; KIESE, 2008). The case study literature shows that not only policies supporting cluster development had an impact on cluster, but also policies with no intended spatial focus. For example, policy measures with unintended spatial effects play an important role for so called high-tech regions, in the early stages of development in particular (STERNBERG, 2003).

The important question is what role does cluster policy play for the development of clusters? Most of the pros in favour of measures supporting the development of clusters can be summed under the concept of systemic and market failure. In addition, the wish for a functioning cluster may be a reason for policy intervention. However, government failures may also lead to a misguided development of the cluster (ROELANDT and DEN HERTOG, 1999).

Thus, in the past decades policies have become more oriented towards the regional level (KOSCHATZKY, 2005). However, they still lack a focus on the different needs of clusters during the life-cycle phases. Cluster policy measures should be adapted to the cultural and political circumstances as well as the cluster's current life-cycle stage (MASKELL and KEBIR, 2006). Blue prints or "one-fits-all"-approaches usually do not lead to the expected results. Nevertheless, the adaptation to regional and cluster characteristics, needs and weaknesses is not always successfully implemented (EUROPEAN COMMISSION, 2008). 
Frequently, policy makers do only consider parts of the cluster concepts when designing cluster policy measures. This partial implementation leaves important connections between the elements of the cluster concept outside the focus (LUNDEQUIST and POWER, 2002). Ignoring the local cultural, historical, and industrial differences, policies run the risk of simply copying other successful clusters.

As mentioned above, the government's impact on the success and competitiveness of clusters is said to be marginal. In a meta-study by VAN DER LINDE (2003) it is shown that there is only minor influence of governmental support on the survival of clusters. Furthermore, this meta-study finds that the number of clusters established by the state is small (although such cases exist). Other concerns about the efficiency of cluster policies relate to the small number of small and medium enterprises that participate in the measures and programs. In addition, many policy programs do not identify market failures. As a consequence, policy measures sometimes tend not to have a real focus. Another challenge is the heterogeneous cluster identification. Cluster policies may be initiated with no real cluster present (HOSPERS and BEUGELDIJK, 2002). This might be one reason explaining the lack of positive results of governmental cluster policies. Additionally, causal relations between the different factors that can be influenced "cannot be determined on the basis of theoretical or philosophical arguments" (JAFFE, 1999, p. 64) but need to be addressed empirically. However, causality may be reversed because highly innovative firms (regions) face less difficulties acquiring R\&D funds (see, e.g. BUSOM, 1999). Furthermore, time lags need to be considered when analyzing outcomes of policy measures (FRITSCH and SLAVTCHEV, 2005).

To sum up, on the one hand, the literature provides arguments that suggest (cluster) policies to be of little importance or to take a marginal, indirect and long-term role, e.g. it is said that policy measures seem to be superfluous because cluster dynamics should make intervention redundant (MASKELL and KEBIR, 2006). On the other hand, the literature 
provides arguments stressing the impact of cluster policy. In case studies, policy is often said to play an important role (BRENNER and MÜHLIG, 2007).

It seems as if the way of policy implementation is crucial for the measure's success. There are many obstacles cluster-related policy has to face. One obstacle we would like to discuss in this paper is the question of how to adjust measures to the needs of the different stages of the cluster life-cycle. In the context of SME policies, a similar topic arises: measures have to be adapted to differences in the way in which innovations are generated and in the importance of innovations (SMALLBONE et al., 2003). In Section II.1 we argue that the stages of the cluster life-cycle differ strongly with respect to the problems and needs that can be addressed by policy. This fact seems to be neglected in policy approaches quite frequently. In Europe, only 36 of 69 national cluster programs have a special focus on clusters in a certain stage of the life-cycle, mainly on the emerging and embryonic phase (EUROPEAN COMMISSION, 2008).

\section{II.3 Measures of cluster policy}

Cluster policy can take many different forms. Above we argued that it is important to find the form which fits the characteristics of the cluster the policy aims at. One of these characteristics is the stage in the cluster life-cycle. In the following, we focus on the various measures that are applied within the framework of cluster policy. We present the instruments and their evaluations if available. We would like to examine the way these instruments work in order to include them in our mathematical model adequately. Furthermore, we would like to review the existing knowledge about the instruments' effectiveness, although there seems to be little knowledge available.

Possible measures to support cluster development are direct and indirect financial support, start-up support, aid for administration, networks and cooperation as well as general assistance for cluster activities. We divide these measures into six categories, bearing in 
mind the measures' impact during the different stages of the cluster life-cycle. These categories are education, public research, $R \& D$ support and influencing the innovation culture, start-up support, network organisation and cooperation support as well as policy measures pointing at the amendment of the infrastructure and local conditions. Most of the time, these measures are implemented by regional or national public authorities. In some cases, however, cluster initiatives are involved. These initiatives aim at similar objectives as "usual" policies (SÖLVELL et al., 2003).

\section{Education}

In the remainder of this paper, cluster policy measures aiming at the amendment of the cluster's knowledge base and skills are subsumed under the label "Education". As knowledge is seen as one of the driving forces of cluster development, "Education" refers to measures aiming at human capital formation (AUDRETSCH and LEHMANN, 2006). These measures, often applied indirectly, help to "create an environment conducive to growth" (CHESHIRE, 2003, p. 332). Policy measures focusing on knowledge creation and skills can be long-term or short-term oriented. Long-term measures address the permanent local conditions, e.g. the set-up of new universities, departments or research institutes. As these set-ups usually involve high investments, they are often implemented in a later stage of the cluster life-cycle, possibly in the maturation stage. However, the timing for such measures is less important than of others (BRENNER, 2008; DTI, 2004).

Short-term measures address local conditions that change in time, e.g. the specific research and lecturing topics at local universities or specific education programs in a region. These measures include specifically qualified education, skill training and staff qualification to improve the knowledge base and skills of the different cluster members in the respective technological field. Furthermore, they are meant to improve the local actors" management skills. 
The development of skills is especially important for the preservation of the cluster in the initial and emerging stages (DTI, 2004). In addition, MARTIN and SUNLEY (2003) point out the importance of linking the cluster to universities in order to profit from the university's research and education fitting the cluster's technological focus. HUGGINS (2008) highlights the importance of training and education not only in the development phases of the cluster. Even later, when the cluster is already in a mature stage, a welleducated workforce and human capital is crucial in order to keep up the cluster's innovative level and avoid a possible decline or lock-in. In this phase, a high level of specialization seems to gain more importance. In Sweden for example, financial support for training, seminars and the exchange of experience is given. The specialization of the cluster's workforce should be promoted in order to increase the economic development in the winning regions (DINNETZ, 2007). Another example is the Life Science Austria Cluster in the Vienna region, which is a cluster in an emerging stage. The measures taken to promote the education of the local workforce are seminars and business plan competitions. In addition, vocational training in the field of business administration for natural scientists, engineers and physicians working in the life sciences is an important measure in these early stages of the life-cycle, because the formation of new companies and start-ups plays an enormous role in these stages. Up to now, these measures have not been evaluated (ENICHLMAIR and OBERHOLZNER, 2007).

To sum up, education plays an important role in cluster development. This seems to hold for all stages of the cluster life-cycle, so that policy measures that improve education should not focus on any stage.

\section{Public research}

Another important aspect which is partly connected with education is "Public research". Public research plays an important role in cluster emergence as it provides specialized 
knowledge and service (PORTER, 1990). But also in a more mature stage, this knowledge is crucial to prevent the cluster from a possible decline. Besides providing knowledge, public research is relevant for the provision and training of a qualified work-force.

Likewise, researchers working at research institutes are potential entrepreneurs as they may found their own company with ideas stemming from the cluster related research (PORTER, 1998b). Therefore, policy makers often regard the implementation of public research as an appropriate measure to promote the economic development of clusters. The establishment of a new research institute is less frequent than the support of research collaborations, a new focus in public research or money for specific projects and additional scientists. Possible measures to reach this aim are, for instance, the establishment of relevant laboratories. Especially in the cluster's initial and emerging phase these may be of relevance. To assure a high product quality right from the beginning, laboratories may be employed for quality verification and certification. But also the development and testing of new products may be sourced out to a specific research institute (FOUNDATION FOR ENTREPRENEURSHIP DEVELOPMENT, 2007). Another possible measure is the implementation of joint industry-research centres that support collaborations between firms and public research institutions. This can be helpful during all stages of the cluster lifecycle (ANDERSSON et al., 2004).

To sum up, public research is often a crucial factor in the development of clusters. Although effects can be expected for all stages of the cluster life-cycle, most arguments focus on the earlier stages.

\section{Supporting R\&D and innovation culture}

The support of public research has to be distinguished from R\&D support and influence on the local innovation culture as those focus on the firm level. The support of R\&D does not 
need to be connected to already existing research institutes but can be implemented in firms only. The innovation culture of a firm may be influenced and thus the innovation culture of a whole region. A well-known example for this is Sophia Antipolis where local and national authorities initiated innovation service activities and thus created an innovative milieu (LONGHI, 1999). This development is mainly important in the early stages of the cluster life-cycle where, on average, the innovation activity is more relevant than in the mature stage. Normally, the impact of innovations decreases with the cluster's maturation. Policy should aim at supporting the cluster's innovativeness by combining key players and qualifications (FROMHOLD-EISEBITH and FROMHOLD, 2005). Formal institutional structures like the establishment of science parks or incubators may be influenced or established by policy and may help to improve the innovation culture and processes (BATHELT and DEWALD, 2008).

In the Cypriotic cluster, for instance, policy put emphasis on these measures. In the cluster's initial stage, in particular, they supported innovations on the production level in order to develop an innovation culture in the region. The program offers financial support to the foremost small and medium sized companies in the targeted clusters in the manufacturing and service sectors. Since the program in Cyprus was started in 2007, there is no evaluation available, yet (PAPADOPOULOS, 2007).

In Germany, the BioRegio-Contest aimed at the overall goal to become one of Europe's largest agglomeration of biotechnology firms. The focus was to enlarge the applicationoriented research in biotechnology and to turn the scientific results into industrial activity by giving financial support to the different projects (HAUSER, 2007). Another example is the South Denmark region with its cluster policy focusing on emerging and developing clusters. The government finances screening activities that should help firms of emerging clusters to address the technological needs in their field of activity. However, access to new technologies is also a measure implemented in more mature clusters when it 
becomes important to gather momentum to prevent the cluster from a possible decline. As the program was launched in 2007 there is no evaluation available, yet (SCHOU, 2007).

To sum up, the support of R\&D activities of firms is mainly assumed to be important in the early stages of the cluster life-cycle. Most of these policy measures address young or emerging clusters. However, it might also be helpful to prevent the decline of existing clusters.

\section{Support of start-ups}

As mentioned above, the support of start-up companies is highly connected with cluster policies since the development of a local firm base is a crucial aspect contributing to the emergence of local clusters. During a phase of technology shift leading to the establishment of networks and structures, start-up companies as well as support organizations are of utmost importance. HUGGINS (2008) argues that in the development phase of knowledge clusters networks and strong ties can lead to a higher number of entrepreneurs and start-up companies in the respective cluster. In later stages, when the cluster constitutes a functioning entrepreneurial base, second-tier start-ups, spin-offs and venture capitalists are needed to sustain the cluster development (WOLFE and GERTLER, 2006).

Therefore, the support and enhancement of start-up companies often plays a significant role in cluster policies. There seems to be no significant difference between the life-cycle phases as measures are applied in the embryonic, emerging and mature phase. The improvement of local conditions trough service firms and other supporting measures should also strengthen the potential for start-ups and spin-offs. In Germany, the BioRegio contest can be taken as an example which aims at enlarging the number of start-up companies in the biotechnology sector through offering financial support. As stated above, 
the ambition was to turn state of the art research knowledge into production activities. The evaluation conducted by the Kiel Institute for the World Economy revealed a positive impact of the BioRegio program, especially on the development of the number of firms. All in all, 600 new companies have been founded; thereof more than 340 in the biomedical sector (HAUSER, 2007).

Likewise, the British government tries to enhance the number of start-up firms in the biotechnology sector by building biotechnology incubators, grow-on facilities and science parks. The overall aim is the transfer of life science research into commercial use and to establish a cluster mainly in the London region. This should be achieved through financial support and training (MAS, 2007).

Finally, the support of the emerging life science cluster in the Vienna region is another example for start-up support in biotechnology clusters in Europe. The measures implemented aim at the use of research for industry applications by providing, for example, business seminars for researchers and by supporting company foundations (ENICHELMEIR and OBERHOLZNER, 2007). The Scottish policy focuses its start-up support on more mature clusters. The ambition is to turn high-end research into commercialisation. An example is the Micro \& Opto Electronics cluster in Scotland, where the focus is on both spin-out and start-up firms. Furthermore, intermediary technology institutes aim at the commercialisation of research in order to support the development of clusters by providing funding for market oriented R\&D. In addition, they can improve local conditions and thus influence the probability of the creation of new firms (MAS, 2007).

Another option is the financing of young and promising firms. However, there are different ways how to organise financial start-up support as well as how this support could affect start-up activities. The applied measures range from the initial to the mature phase as young innovative companies are important in every developmental phase of a cluster (WOLFE and GERTLER, 2006). In the London Biotechnology Cluster, the establishment 
and support of a seed fund is used to prevent young firms from financing shortfalls, especially in the embryonic cluster phase when venture capital firms do not exist, yet (MAS, 2007). The Estonian government, for example, puts a stronger focus on supporting SME by offering state guarantees for loans, leases and bank guarantees as well as traditional venture capital funds for operating and fast growing small companies. This approach is especially implemented in the emerging phase of clusters. In contrast, in Sweden the establishment of venture capital and services, e.g. business angels, is used as a measure to guarantee the number of firms in a mature cluster (DINNETZ, 2007). To sum up, policy measures that aim at increasing the regional start-up activity are applied to clusters in all stages of their life-cycle.

\section{Network organisation and cooperation support}

Quite often, network policy and cluster policy are treated synonymous; especially by policy makers. Frequently, policy makers support regional network organisation or cooperation and call these measures cluster policy. However, networks are only one part of the complex structure and dynamics of clusters; focusing only on this part does not satisfy the holistic cluster approach (KIESE, 2008). Hence, we argue that the support of network organisations and cooperation is one way among many others to support clusters.

The support of network organisation and cooperation seems to be less important in later stages of the cluster life-cycle. The focus on network organisation is more relevant in the initial and expansion phase when it becomes important to establish well functioning networks and cooperation between the different actors in the cluster. In these stages, the actors are often technologically scattered; furthermore, the uncertainty about the cluster's development is high. Thus, networks represent a possibility to minimize risks. Even in the growing phase networking plays an essential role to prevent isolation and help to generate 
a positive environment. In this phase, cluster organisations and networks supporting the local infrastructure help to address the needs of the cluster members (MENZEL and FORNAHL, 2007). HUGGINS (2008) also highlights the significance of networks for the development of clusters, which are in these early stages mainly informal with frequent contacts and strong ties. When becoming a growing cluster, the mode of interaction changes to more formalized networks and cooperation, which have established during the initial phases. However, network support is also of some significance in later stages of the cluster life-cycle when networks become mature, inducing an endangerment of overembeddedness and lock-in.

It can be distinguished between inter-firm cooperation, cooperation between firms and public research institutes, and overall networking in a cluster. The main concern is to support already existing regional activities and, thus, to stimulate emerging networks and cooperation. The Austrian government supports networks and cooperation in various ways (ENICHELMAIR and OBERHOLZNER, 2007). Mainly in the embryonic phase, the government tries to enhance networking in the life-science cluster in the Vienna region by creating contacts between the different members like co-operation partners and financiers. In Lower Austria, the integration of large firms and SME in co-operation networks should help to increase the innovativeness of the involved firms. In the first case, the focus is on networks including all members of the cluster whereas in the second case only inter-firm networks are promoted. But also cooperation between research and industry are supported in order to strengthen high-end research in a certain field by providing industry coordinators.

Denmark's cluster policy focuses on emerging networks (SCHOU, 2007). A joint secretariat should help to coordinate collective activities, such as knowledge exchange, and function as a connection between firms, institutes and education set-ups. Thus, the focus is on general networks for all cluster members. Other possible measures supporting clusters in 
the early stages of the life-cycle are conferences, meetings and other regional learning processes. Facilitators should help to connect the different cluster members and create networks between them. No evaluation has been carried out yet, but different positive developments concerning the cooperation between firms have been observed.

As networks and cooperation grow older, there are some policies aiming at the renewal of networks and cooperation in later stages of the cluster life-cycle. Over-embeddedness and a possible lock-in in a mature stage should be prevented by developing and mobilizing human capital and new linkages between actors. New modes of interaction can help to renew deadlocked networks and cooperation (HUGGINS, 2008; RAINES, 2000). The Spanish cluster policy focuses on the renewal of networks, communication and collective identity in order to support mature clusters. For this purpose, the development of weak or even non-existing ties in the value chain brings new options for collaborations and network partner into the cluster. This is implemented by financial support for common projects of the cluster members as well as the creation of physical space for cooperation (MÜLLER, 2007).

A widespread problem of cluster policies aiming at the development of networks is the strong inward-looking focus. This does not imply that the internal cohesiveness of a cluster is of no importance; however, outward-looking networks and communication are crucial to bring new ideas into the cluster. Nevertheless, these outward-oriented networks are often neglected (BATHELT et al., 2004).

To sum up, the support of regional networking and cooperation is mainly done in the earlier stage of the cluster life-cycle. However, there are also policy measures focussing on networks and cooperation which are applied in mature stages aiming at the cluster's renewal. 


\section{Infrastructure and local conditions}

Infrastructure and local conditions are in general seen as important factors for the economic development of a region. As the cluster grows, the qualitative and quantitative needs in a region change and require adjustment and improvement. Often, local conditions fulfil the needs for a cluster's emergence but not for its growth. Therefore the improvement of these conditions is important in emerging and growth phases (MENZEL and FORNAHL, 2007). However, if a cluster becomes mature and successful, large infrastructure investments become more likely. If a cluster is still in a developing phase, the set-up of airports, highways or new universities and research institutes is attended by uncertainty. It needs a strong lobby which is more likely to be found in a mature cluster with strong ties between the different actors (BRENNER, 2008). Nevertheless, no empirical examples have been found.

\section{Summary}

Table 1 lists all measures including the different stages the measure is more likely to succeed. Obviously, for some measures it is difficult to distinguish between the different phases.

Table 1: Policy measures and the different stages the measure is more likely to succeed

III. Model

As stated above, there is only little empirical evidence for the effects of different policy measures supporting clusters so far. If evaluations of conducted programs do exist, they neither compare different approaches nor do they examine the effects with respect to the cluster's current stage of development. We expect more empirical evidence to be collected 
in the future. However, at the moment, a theoretical approach seems to be more helpful.

The idea behind our theoretical approach is to develop a mathematical model of industryspecific regional development and then to use this model to deduce knowledge about the impact of different policy measures conducted at different points in time. First, we give a verbal description and discussion of the model (Section III.1). In Section III.2 the mathematical formulas describing our model are given. The estimation of the model parameters is described and discussed in Section III.3.

Our approach proceeds as follows. We build a model that describes the regional industryspecific developments in general (Sections III.1, III.2 and III.3). Then, we determine how this model differs with respect to the various stages of the cluster life-cycle (Section III.4). Finally, we define a number of potential policy measures, implement their direct impact within the model, and study their effect on regional development (Section IV).

\section{III.1 Basic considerations and model structure}

In a first step, we set up a model that describes regional industry-specific development. Of course, such a model can be set up in an arbitrarily complex way including a large number of variables. We persue two aims that influence the kind of model we develop. First, the model should be as simple as possible in order to conduct a comprehensive analysis of the effects of the different policy measures. Second, the model's parameters and mathematical formulas are empirically estimated as far as possible to ensure the model's reliability.

This has two implications for the variables used in our model. First, the number of variables should be kept as small as possible. Second, variables should only be used if the variable itself, their dynamics, and their interaction with other variables can be studied 
empirically.

Two kinds of variables have to be distinguished. On the one hand, there are endogenous variables, i.e. variables whose dynamic can be described by all other variables. On the other hand, there are exogenous variables. Their impact on the endogenous variables is implemented in the model, but no causal dependence in the opposite direction is considered. Of course, in reality all variables are endogenous. In theoretical models, the number of endogenous variables determines whether a model is analytically treatable while the number of exogenous variable can be as large as the modeller wishes. Thus, the endogenous variables have to be chosen with care.

We want to model the development of clusters. The variables that are used commonly in the literature to identify clusters empirically are the location quotient and the number of employees (SFORZI, 1990, STERNBERG and LITZENBERGER, 2004, BRENNER, 2006). The location quotient is an index based on the employment numbers. Hence, the number of employees seems to be an adequate variable to measure the development of clusters and the success of policy measures, although policy makers might aim at more than employment growth. Employment is the central endogenous variable in our model.

Other variables that play a prominent role in the cluster literature are the number of firms, start-ups, innovations, networks, human capital, spillovers, venture capital and many more (see BRENNER and MÜHLIG, 2007 for a comprehensive list of factors and mechanisms). In our model, we include the number of start-ups, the number of innovations and the strength of networking as further endogenous variables. This is a subjective choice that is partly driven by the availability of data and partly by our evaluation of the variables' importance. Some aspects, such as human capital and spillovers, are somehow implicitly integrated in the model. As mentioned above, we have to neglect some variables. Our choice of variables can - to a certain degree - be justified by the findings of BRENNER (2001). According to Brenner, for the fundamental characteristics of cluster models it does 
not matter whether only one or more self-augmenting processes are included.

Hence, our model consists of one central endogenous variable, employment, three further endogenous variables, start-ups, innovations and networks, and a number of exogenous variables, education, public research, market conditions, population, governmental start-up support, innovation support, network support and local infrastructure. The model's structure is depicted in Figure 1.

Figure 1: Structure of the mathematical model

As stated above, the model does not include all interactions that are relevant for the development of clusters. First, we have restricted the model to a small number of endogenous variables. Second, we only consider further variables that correspond with our basic categories of policy measures that we defined above. However, we believe that the most important aspects are included. The strength of the model is that it can be empirically validated, at least in its main elements.

III.2 Mathematical formulas of the model

Above, all variables of the model have been defined and discussed. In the mathematical formulation we use the following abbreviations:

- $e_{t}$ : employment

- $i_{t}$ : number of innovations

- $s_{t}$ : number of start-ups

- $x_{t}$ : intensity of network activities

- $r_{t}$ : public research expenditure 
- $I_{t}$ : amount of qualified labour

- $\quad p_{t}$ : population (number of inhabitants)

- $m_{t}$ : market conditions

- $f_{f, t}$ : local infrastructure

- $f_{s, t:}$ start-up support

- $f_{x, t:}$ network support

- $f_{i, t}$ : innovation support

In order to keep the analysis as simple as possible and make the mathematical model treatable, we do not consider interdependencies between regions. Hence, the model reflects the development within one cluster. The cluster's economic environment is considered only within the variable $m_{t}$, reflecting the global market situation. This implies that all other variables refer to the region and the industries involved in the cluster under consideration.

As stated above, the development in time is explicitly modelled only for the endogenous variable. In the following, we set up the dynamic equations for these four variables.

\section{Employment}

Empirical examinations prove hat the dynamics of regional employment show the same characteristics as the growth of firms (SCHLUMP and BRENNER, 2010). They are best modelled on logarithm scale and by defining an attraction value for the employment number, $\hat{e}_{t}$, towards which the change of employment converges:

$$
\ln \left(e_{t+1}\right)=\ln \left(e_{t}\right)+\ln \left(\frac{\hat{e}_{t}}{e_{t}}\right)+u_{e}
$$

$u$ is the usual error term that reflects the randomness of the dynamics. SCHLUMP and 
BRENNER (2010) find that the attraction value, $\hat{e}_{t}$, depends on various variables that characterise the regional situation. For some of these variables the dependence is additive, while for some variables it is multiplicative. For each variable influencing the change of employment we have examined empirically whether the variable should enter the equation above additively or multiplicatively (for detailed explanations of the approach see SCHLUMP and BRENNER, 2010). As a result, we model $\hat{e}_{t}$ by

$$
\hat{e}_{t}=\left(\gamma_{e, e} \cdot e_{t}+\gamma_{e, s} \cdot s_{t}+\gamma_{e, l} \cdot l_{t}+\gamma_{e, r} \cdot r_{t}+\gamma_{e, f} \cdot f_{f, t}\right) \cdot L Q_{t}^{\gamma_{e, q}} \cdot m_{t}^{\gamma_{e, m}} \cdot i_{t}^{\gamma_{e, i}} \cdot x_{t}^{\gamma_{e, x}} \cdot\left(\frac{p_{t}-e_{t}}{p_{t}}\right)^{\gamma_{e, p}}
$$

where $L Q$ stands for the location quotient which is defined as usual by

$$
L Q_{t}=\frac{e_{t} \cdot E_{g l o b, t}}{e_{g l o b, t} \cdot E_{t}}
$$

"glob" signifies that a variable relates to the whole world, $E$ is the employment in all industries and the $y$ 's stand for various parameters that determine the strength of the relationship between the various variables and employment.

\section{Start-ups}

In order to model the number of start-ups we use the approach of BRENNER and FORNAHL (2008) in which the number of start-ups is modelled as a binomial distribution with the number $\hat{n}_{s, t}$ of potential founders given by

$$
\hat{n}_{s, t}=\varphi_{s, p} \cdot p_{t}+\varphi_{s, e} \cdot e_{t}+\varphi_{s, l} \cdot l_{t}+\varphi_{s, r} \cdot r_{t}
$$

and the probability $P_{s, t}$ that each of the potential founders really starts a firm given by

$$
P_{s, t}=\frac{1}{1+\exp \left[\varphi_{s, 0}-\varphi_{s, f} \cdot f_{s, t}\right]} .
$$

All $\varphi$ are parameters that we estimated empirically (see Section III.3). The way in which 
the variables are included in Equations (3) and (4) is extensively discussed and estimated empirically by BRENNER and FORNAHL (2008).

\section{Innovations}

To model innovations we use the same approach as for the number of start-ups and distinguish between potential innovators and their probability to innovate (for a detailed discussion of this distinction see BRENNER and BRÖKEL, 2009). We denote the number of potential innovators by $\hat{n}_{i, t}$. We tested empirically a number of different variables, e.g. research institutes and universities. We found that only two variables significantly influence the number of potential founders: the population $p_{t}$ and the employment $e_{t}$. The employment has a much stronger impact on the regional start-up activity. Neglecting the population's impact, the later analysis can be simplified tremendously, so that we write:

$$
\hat{n}_{i, t}=v_{i, e} \cdot e_{t} .
$$

All other variables influence the probability $P_{i, t}$ that these potential innovators conduct an innovation in a given year. This probability is found to be given by

$$
P_{i, t}=\frac{1}{1+\exp \left[v_{i, 0}-\left(v_{i, r} \cdot r_{t}+v_{i, f} \cdot f_{i, t}\right)\right]} .
$$

All $v$ in Equations (5) and (6) are parameters.

\section{Networking}

In contrast to employment, start-ups and innovations, there are no empirical studies available yet that illustrate the interrelation between the intensity of local networking and other local variables. We assume a similar structure as for start-ups and innovations. Again, the more employees are present in a region, the more network activity might 
develop. However, this increase in network activity might be non-linear. Since we are not able to check this assumption empirically, we choose a flexible formulation. In addition, the network intensity depends on the some local characteristics and the network support present. We define, without being able to test this empirically:

$$
x_{t}=\frac{v_{x, e} \cdot e_{t}^{\gamma_{x, e}}}{1+\exp \left[v_{x, 0}-v_{x, f} \cdot f_{x, t}\right]}+u_{x} .
$$

Again, all $v$ are parameters and $u$ is the usual error term.

\section{Limitations of the model}

There are two main limitations of the model. First, we only include a number of variables for most of which empirical data is available so that we can estimate the relationships reflected in the model. A number of potentially influential variables are ignored. The variables that are most frequently assumed to be important in the literature are included. However, industries differ and the various variables are of different importance in the industries. Therefore, there might be industries whose main mechanisms cannot be represented by our model. In theoretical literature it is usually argued that the exact mechanisms do not matter. Often local externalities are included in a quite sketchy manner. BRENNER (2001) provides evidence that a detailed modelling of all relevant processes might, indeed, not be of crucial importance. From this, we might conclude that the dynamics of cluster formation are well reflected by our model, although not all details are included in the model.

The same does not hold for the impact of policy measure. The model only allows us to examine those policy measures for which the way they influence the regional development is explicitly included in the model. Policy measures can be designed in many different ways. Thus, they have distinct impact on the different aspects of the complex development 
of clusters. Not all of them are reflected in the model. Above, we categorised the potential policy measures. Each category does summarise a number of different activities and mechanisms. In most cases, only one mechanism is implemented in the model as representative of the category. This implies that not all measures are represented adequately. Therefore, we are only able to draw reliable conclusions for those measures implemented in our model.

Second, we only consider one industry and one region at a time. This simplifies the model tremendously in order to make a mathematical analysis possible. However, this implies that we do not model interrelations between industries and between regions. Part of the interregional interrelations is reflected in the market situation, $m_{t}$. Furthermore, a number of variables that represent the situation in the region are included in the model. If neighbouring regions have an impact on the regional developments, we might represent these impacts by those variables. Hence, from a mathematical perspective, it makes no difference whether we include other regions and industries or not as long as the interrelations with other regions and industries do not lead to endogenous dynamics. Furthermore, in a theoretical approach we do not have to define industries and regions beforehand. We are able to declare that all activities that belong to the cluster examined, irrespective of industrial classifications and regional borders, are included in the analysis. This is, of course, not possible in the calibration of the model using the empirical data below.

\section{III.3 Model validation and calibration}

Above, we have argued that the results of our modelling approach are only reliable if the model is validated and calibrated using empirical data. Three topics have to be addressed.

First, we have to find the adequate variables that should be included in the model. This 
topic is discussed above.

Second, the model has to represent the interrelations between these variables adequately. This means the functional form of the interrelations has to match reality. As stated in Section III.2, we used findings from previous studies and conducted additional empirical tests in order to find out whether the variables influence each other in additive or multiplicative ways. For all variables that we have empirical proxies for the above equations represent the form that fits reality best. An exception is the network variable, $x_{t}$. For this variable, we do not have adequate data so that the equation for this variable is much less reliable.

Third, the model has to be calibrated. This means that the parameters of the model have to represent reality adequately. For this calibration we use data for the German labour market regions for the time period from 1999 to 2007. For most of the variables included in the model we have exact data or, at least, some proxies. The data sources are the German Statistical Office, the German Patent Office, and the German Institute for Labour. We have data on the employment, $e_{t}$, the number of start-ups, $s_{t}$, and the number of inhabitants, $p_{i}$. For the number of innovations, $i_{t}$, we use the number of patents as a proxy. Qualified labour, $I_{t}$, is approximated by the number of university graduates; public research expenditures, $r_{t}$, are approximated by the number of employees in public research institutes.

The idea behind the model's calibration is not to obtain exact values for the parameters, but to ensure the size of the parameters is approximately correct. The aim is to obtain a reliable model and not to estimate effects empirically (for such estimations see BRENNER and FORNAHL, 2008; SCHLUMP and BRENNER, 2010). Therefore, the use of proxies seems to be adequate.

Anyhow, the parameters differ between industries. One correct set of parameters does not exist. Hence, we conduct the calibration of the model for a number of industries 
separately: textiles, chemicals, metal, machinery, automotive, electrical and instruments. We decided to use these industries since there does exist a good concordance between the industries' classification and the respective patent classes. This provides us with knowledge about the parameter values as well as some insights about whether they depend on the stage of the industrial life-cycle. The median estimates of the parameters are presented in Table A. 1 in the appendix. If we can observe a clear dependence of a parameter on the age of the industry, we state this also in Table A.1.

The parameter values are, of course, crucial for the results we obtain below. We use the median values estimated for a number of industries. This implies that not all industries are represented adequately by the resulting model. Therefore, we have to discuss how the results change if the parameters are different. Furthermore, some industries might possess specific features and, thus, exhibit extreme values for some parameters. Hence, we can never be sure that our results hold for all industries. Since the industrial and regional classifications used in the empirical analysis might not be adequate, the aim of the analysis can only be to find some tendencies describing the effectiveness of the various policy measures that hold for the average industry. For this reason, we do not use the empirical obtained parameters as exact values but to give us an impression about the approximate size of the parameters. However, it is important to know, at least, something about the values of the parameters; otherwise we might deduce arbitrary results from the model. Robustness checks show some evidence that the results are likely to hold for most industries.

III.4 Modelling the stages of the cluster life-cycle

The model above describes the regional employment dynamics in relation to a number of other variables. Thus, it describes the emergence and development of clusters if we define a cluster basically as an agglomeration of specific economic activity in a region. Many of 
the mechanisms assumed to be important for the development of clusters are included in the model above.

The importance of these mechanisms changes during the different stages of the cluster life-cycle. The aim of this paper is to study how these changes influence the effectiveness of different policy measures. In the model, a change in the mechanisms' importance is reflected by a change of the parameters' values. Hence, to understand the differences between the distinct stages of the cluster life-cycle by means of the model we have to know how the parameters differ between these stages.

There are two sources for obtaining information on these differences: the theoretical discussion of the cluster life-cycle as presented in Section II.1 and empirical estimations of the parameters for different industries. Table A.1 lists the parameters and their changes during the cluster life-cycle that are predicted on the base of different theories and empirical estimations.

Of course, clusters do not jump from one stage to another. As discussed above, there are transition phases. Similarly, the parameter values should not be expected to jump from one value to another. While the cluster transits from one stage to another, we rather expect them to continually decrease or increase. However, we are mainly interested in the effectiveness of policy measures within the different stages. Thus, the analysis of the model focuses on the three main stages of the cluster life-cycle.

\section{Analysis, results and discussion}

\section{IV.1 Analysis of the model}

In order to analyse the model, we rewrite Equations (3), (4), (5) and (6) such, that they have a similar structure as Equations (2) and (7): 


$$
s_{t}=\frac{\varphi_{s, p} \cdot p_{t}+\varphi_{s, e} \cdot e_{t}+\varphi_{s, l} \cdot l_{t}+\varphi_{s, r} \cdot r_{t}}{1+\exp \left[\varphi_{s, 0}-\varphi_{s, f} \cdot f_{s, t}\right]}+u_{s}
$$

and

$$
\left.i_{t}=\frac{v_{i, e} \cdot e_{t}}{1+\exp \left[v_{i, 0}-\left(v_{i, r} \cdot r_{t}+v_{i, f} \cdot f_{i, t}\right)\right.}\right]+u_{i}
$$

In these two equations, the error terms are neither symmetrically distributed nor they are independent from the values of the parameters. However, this transformation allows to insert Equations (7), (8) and (9) into Equation (2).

In the resulting equation, the error terms are quite complex and a mathematical treatment of the model including these error terms is not possible. The aim of our mathematical analysis is to examine the influence of the parameters and exogenous variables on the dynamics. We are not able to fit the parameters and model real developments exactly. However, we are interested in the direction of the parameters' impacts. For this aim, an analysis of the model excluding the error terms is sufficient.

Ignoring the error terms, we obtain the still quite complex Equation (A.1), which is given in the appendix. Equation (A.1) contains terms that depend on employment $e_{t}$ and terms that depend only on the exogenous variables and the parameters. For the analysis of the dynamics of $e_{t}$ the terms depending on variable $e_{t}$ are of special importance. They determine the structure of the dynamics. Therefore we separate these two kinds of terms and use abbreviations in Equations (A.2), (A.3), (A.4) and (A.5) for the terms that do not include $e_{t}$. We obtain

$$
\log \left(e_{t+1}\right)-\log \left(e_{t}\right)=\log \left[c_{t} \cdot\left(a_{t} \cdot e_{t}^{\alpha}+b_{t} \cdot e_{t}^{\alpha-1}\right) \cdot\left(\frac{p_{t}-e_{t}}{p_{t}}\right)^{\gamma_{e, p}}\right]
$$

Let us first examine the term within the logarithmic function on the right-hand side of Equation (10): 


$$
L\left(e_{t}\right)=c_{t} \cdot\left(a_{t} \cdot e_{t}^{\alpha}+b_{t} \cdot e_{t}^{\alpha-1}\right) \cdot\left(\frac{p_{t}-e_{t}}{p_{t}}\right)^{\gamma_{e, p}}
$$

This term shows a clear dependence on the number of employment $e_{t}$. For $L\left(e_{i}\right)$, we obtain a functional form that looks as depicted in Figure 1.

For further mathematical treatment, we use the following assumption:

Assumption 1: Custer forces play a role for the cluster's development. Mathematically, this is given if the last term in Equation (10) plays no role for small values of $e_{t}$ and the term $b_{t} \cdot e_{t}^{\alpha-1}$ plays no role for large values of $e_{t}$

Figure 2: Function $L\left(e_{t}\right)$ for the median parameter values and a region with 800,000 inhabitants.

Assumption 1 allows us to distinguish between the case of low and medium employment, for which negative local externalities play no role, and the case of high employment, for which the dynamics are determined by cluster forces and negative externalities only. In the appendix, we prove the following results:

Proposition 1: If Assumption 1 is satisfied, the local system shows one of the following three dynamics:

A) The system has one stable state that is characterised by a low number of employment. The system converges to this sole stable state. We find such a dynamic if

$$
c_{t} \cdot a_{t} \cdot p_{t}^{\alpha} \cdot \frac{\gamma_{e, p}^{\alpha}+\alpha^{\gamma_{e, p}}}{\left(\gamma_{e, p}+\alpha\right)^{\alpha}}<1
$$


is satisfied.

B) The system has two stable states of which one is characterised by a low number of employment and the other by a high number of employment. The system converges to one of these two stable states depending on the state the system is currently in. We find such a dynamic if $\alpha<1$,

$$
\begin{gathered}
c_{t} \cdot b_{t}^{\alpha} \cdot a_{t}^{1-\alpha} \cdot\left[\left(\frac{1-\alpha}{\alpha}\right)^{\alpha}+\left(\frac{\alpha}{1-\alpha}\right)^{1-\alpha}\right]<1 \quad \text { and } \\
c_{t} \cdot a_{t} \cdot p_{t}^{\alpha} \cdot \frac{\gamma_{e, p}^{\alpha}+\alpha^{\gamma_{e, p}}}{\left(\gamma_{e, p}+\alpha\right)^{\alpha}}>1
\end{gathered}
$$

or if $\alpha>1$ and Condition (8B2) is satisfied.

C) The system has one stable state that is characterised by a high number of employment. The system converges to this sole stable state. We find such a dynamics if $\alpha<1$ and

$$
c_{t} \cdot b_{t}^{\alpha} \cdot a_{t}^{1-\alpha} \cdot\left[\left(\frac{1-\alpha}{\alpha}\right)^{\alpha}+\left(\frac{\alpha}{1-\alpha}\right)^{1-\alpha}\right]>1
$$

Proposition 1 means that, depending on the model's parameters, we find one of three possible dynamics in a region. The first possible dynamic $(A)$ predicts that no cluster will emerge or an existing cluster will disappear. The second possible dynamic (B) predicts that if the critical mass is exceeded, a cluster will emerge or stabilise, while no cluster will appear as long as the regional economic activity is below the critical mass. The third possible dynamic $(\mathrm{C})$ predicts that a cluster will emerge or stabilise irrespective of the current regional activity. The parameters of the model determine what kind of dynamics will occur. Moreover, as argued above, the parameters of the model change during the cluster life-cycle and can be influenced by various policy measures. These are the impacts we 
want to analyse.

Before examining the impact of policy measures and the different stages of the cluster lifecycle, let us check the relevance of Proposition 1 for the median case. If the market situation is average, i.e. demand and supply are in an equilibrium $\left(m_{t}=1\right)$, a region with no university and no relevant public research institute falls into category B if it has many inhabitants (more than approximately 550000); if a region has not that many inhabitants, it falls into category A. The former case is depicted in Figure 1 with the two stable states marked by a star. Hence, for an average industry, both situations, clustering and no clustering, are stable in large regions.

Category $C$ is found if the local positive externalities (given by $\alpha$ and $a_{t}$ ) are of high intensity or the market conditions are favourable (high value of $m_{t}$ ). In addition, positive local circumstances that influence the value of $b_{t}$ make category $\mathrm{C}$ more likely but have a much smaller impact than the other variables depicted above. However, category $C$ is not a standard case, which implies that clusters do not emerge everywhere at all times.

\section{IV.2 Theoretical impact of policy measures}

The model above allows us to check the impact of various policy measures on the dynamics of the local system during different phases of the cluster life-cycle. We proceed in three steps. First, we use the mathematical findings of Section IV.1 in order to identify the kind of dynamics likely to occur in the three stages of the cluster life-cycle. Furthermore, we discuss to what extent these dynamics are influenced by the values of the terms $a_{t}, b_{t}$ and $c_{t}$ in Equation (10). Second, we list the relevance of each policy measure means in terms of the mathematical model and how these policy measures influence the terms $a_{t}, b_{t}$ and $c_{t}$. Third, we combine the results from the first two steps and analyse the policy measures' impact in the three phases of the cluster life-cycle (Section 
IV.3).

\section{Dynamics in the three stages of the cluster life-cycle}

Above, we found that three kinds of dynamics are possible: Dynamics A, B and C. We also found that for the median parameters we rather have to expect Dynamics A or B. However, there are differences between industries and, as discussed above, the parameters change during the cluster life-cycle. These changes are listed in Table A.1 in the appendix. In the following we use this knowledge in order to find out whether certain kinds of dynamics are more likely to occur in certain stages of the cluster life-cycle.

Above we argued that we cannot be sure about the values of the parameters. They differ between industries and empirical estimates only give some crude knowledge about the parameters' size. We will consider this in the analysis below. This means, that we only use the estimated parameters to get a feeling for the most likely situation. However, we will also discuss the implications for parameters deviating from these values.

Before we examine the different stages, we discuss what conclusions can be drawn for the expected dynamics from the values of the terms $\alpha, a_{t}, b_{t}$ and $c_{t}$. Proposition 1 tells us that

- Dynamic $A$ is expected if $a_{t}$ and $c_{t}$ are small.

- Dynamic B is expected either if $a_{t}$ and $c_{t}$ are large and $\alpha$ is bigger than 1 or if $b_{t}$ is small.

- Dynamic $\mathrm{C}$ is expected if $a_{t}, b_{t}$ and $c_{t}$ are large and $\alpha$ is smaller than 1.

Now, we discuss what the parameter values listed in Table A.1 imply for the terms $\alpha, a_{t}, b_{t}$ and $c_{t}$ for the different stages of the cluster life-cycle. We discuss each stage separately (a summary is given in Table A.2 in the appendix):

- Initial phase: The term a depends on parameters that, in the initial phase, are either 
small or medium. Hence, $\alpha$ is small and definitely below 1 in this phase. The parameters that determine $a_{t}$ are either small or very large. We expect a medium value of $a_{t}$, although this is not as clear. Many parameters are involved in the term $b_{t .}$ In the initial phase, all of them are large or very large so that we expect a very large value of $b_{t}$. The parameters that determine term $c_{t}$ are medium or small so that we expect a small value for $c_{t}$. These values imply that Dynamic B are not possible or, at least, very unlikely. Most likely we will see Dynamic C and there is some chance to find Dynamic A.

- Expansion phase: In this phase, all parameters that determine the value of $\alpha$ are either large or very large. Hence, $\alpha$ is very large in this phase. However, $\alpha$ is, on average, 0.5 so that it is likely that $\alpha$ is above 1 in this phase. The term $a_{t}$ can be expected to be very large because all crucial parameters are large or very large. Most of the values involved in term $b_{t}$ are either large or very large in the expansion phase. However, one parameter is medium. Thus, we expect the term $b_{t}$ to be large but this is less clear. Almost all parameters that determine term $c_{t}$ are very large so $c_{t}$ should also be very large. These expectations for the terms $\alpha, a_{t}, b_{t}$ and $c_{t}$ make Dynamic A impossible or, at least, very unlikely. It is unclear whether Dynamics B or C should be observed.

- Mature phase: The parameters that determine the value of $\alpha$ range between medium and very large. Hence, a can be expected to be large but it has to be doubted whether it is greater than 1 . The term $a_{t}$ has a rather small value since the parameters involved are between small and medium in this phase. Term $b_{t}$ is expected to take a small value because all parameters involved are either small or medium in the mature phase. In this phase, the parameters involved in term $c_{t}$ vary in their size. Most are medium but some are very large. Hence, the value of $c_{t}$ is less clear with an expectation between medium and large. It is less clear what kind 
of dynamics should be expected here. The value of $a_{t}$ implies rather Dynamic $A$. However, the value of $c_{t}$ might lead to Dynamics B or $\mathrm{C}$, while a small value of $b_{t}$ makes Dynamic B more likely.

The arguments above are summarised in Table 2.

Table 2 Model implications for the dynamics in the three stages of the cluster life-cycle.

\section{Mathematical meaning and impact of the various policy measures}

It is important to list the implementation of the various policy measures in the model in detail. As stated above, each of our policy measure categories contain many different approaches that are likely to impact different variables and processes. In the mathematical model, we implemented each category in exactly one way. Hence, from the analysis below, we only obtain reliable information about the impact of those policy measures that work exactly the way they are implemented in the model.

Hence, in the following, we discuss three topics for each policy measure category: 1) which variable is used to represent the measure in the model, 2) which real effects and processes are reflected, and 3) how does this impact on Equation (10).

- Education: Education is reflected in the model by the amount of highly educated people, $I_{t}$, (people with university degree). In the model, this is assumed to have an impact on the number of start-ups and the growth of firms. However, in the model, education is implemented as an exogenous variable which implies that learning on the job is not represented. Improving or increasing education implies: $I_{t} \uparrow \rightarrow b_{t} \uparrow$, especially if $\gamma_{e, l,} \gamma_{e, s}$ and $\gamma_{s, l}$ are large. 
- Public research: The amount of relevant public research in the region is directly included in the model by the variable $r_{t}$. It is assumed that this amount has an impact on the number of start-ups, innovations and the growth of firms. Increasing public research implies: $r_{t} \uparrow \rightarrow b_{t} \uparrow$, especially if $\gamma_{e, r}, V_{e, s}$ and $\gamma_{s, r}$ are large and $c_{t} \uparrow$, especially if $\gamma_{e, i}, v_{i, e}$ and $v_{i, r}$ are large.

- Supporting R\&D and innovation culture: The model contains a variable, $f_{i, t}$, which reflects any support from the outside that increases the innovativeness of firms. Other effects are not assigned to this variable. Hence, all measures that only increase innovativeness are well represented by this variable. Increasing innovativeness implies: $f_{i, t} \uparrow \rightarrow c_{t} \uparrow$, especially if $\gamma_{e, i}, v_{i, e}$ and $v_{i, f}$ are large.

- Support of start-ups: The model contains a variable, $f_{s, t}$, which reflects any support from the outside that increases the regional start-up rate. Other effects are not assigned to this variable. Hence, all measures that support start-up activities and, thus, make them more likely are well represented by this variable. Increasing startup activity implies: $f_{s, t} \uparrow \rightarrow a_{t} \uparrow$, especially if $\gamma_{e, s}, \varphi_{s, e}$ and $\varphi_{s, f}$ are large and $b_{t} \uparrow$, especially if $\varphi_{s, f}$ is large.

- Network organisation and cooperation support: The model contains a variable, $f_{x, t}$, which reflects any support from the outside that increases the regional network activities. Other effects are not assigned to this variable. Furthermore, the model includes only effects of networks on the growth of firms. Hence, all measures that support network activities and, thus, help firms to grow are well represented by this variable. If network support increases the start-up activity or innovativeness, such an effect is represented in the by the two variables, $f_{i, t}$ and $f_{s, t}$. Increasing network activity implies: $f_{x, t} \uparrow \rightarrow c_{t} \uparrow$, especially if $\gamma_{e, x}, v_{x, e}$ and $v_{x, f}$ are large.

- Infrastructure and local conditions: The model contains a variable, $f_{e, t}$, which reflects any support from the outside that supports the growth of firms. All direct help to 
firms can be subsumed under this label. Again, only this direct effect is reflected in the model. If a policy measure has additional effects, other variable changes have to be considered simultaneously. Increasing support of firms implies: $f_{e, t} \uparrow \rightarrow b_{t} \uparrow$, especially if $Y_{e, f}$ is large.

\section{IV.3 Impact of policy measures in the three stages}

In the following, we discuss how the different policy measures outlined above influence the cluster processes for each stage separately. Interpreting the model's result, it has to be kept in mind that we are only able to make statements about policy measures that are applied in the form described above.

\section{Initial phase}

According to the findings summarised in Table 1, the question in the initial phase is whether a cluster emerges or not. In this phase, policy measures could be applied in order to increase the probability of cluster emergence. This would imply that in the model the likelihood of Dynamics C should increase and the likelihood of Dynamics A decreased. According to Table 1, this is reached by measures that increase $a_{t}$ and/or $c_{t} . b_{t}$ does not play a role.

According to the findings in Section IV.2, education, infrastructure and local conditions do only influence the value of $b_{t}$ but not the values of $a_{t}$ and $c_{t}$. Hence, measures that aim at education or infrastructure, and local conditions should not be expected to have an impact in the initial phase of cluster development. They do not increase the likelihood of cluster emergence.

In this context, we have to keep in mind that the above findings only hold for policy 
measure that work as modelled here. This means that educational measures influencing the growth of firms only have no impact in the initial phase. For example, educational measures that increase regional start-up activity are not modelled explicitly in this context and are subsumed in the support of start-up activity. Hence, for such educational measures the statement above does not hold.

Public research is found to increase the term $c_{t}$ so that the emergence of clusters becomes more likely in regions in which the government conducts public research. According to the findings above, this holds especially if $\gamma_{e, i}, v_{i, e}$ and $v_{i, r}$ are large. If innovations play an important role in the considered industry, the first two parameters are large. The latter parameter, $v_{i, r}$, is large if public research strongly contributes to the regional innovation activity. Hence, in innovative industries, in which public research contributes significantly to innovation activity, policy measures that support public research in the initial phase of the cluster life-cycle increase the probability of cluster emergence.

Similar results are obtained for the support of private $R \& D$ activities or the support of a regional innovative culture. Again, such support increases the likelihood of cluster emergence. This is especially the case if innovations are important in the industry under discussion (large $V_{e, i}$ and $v_{i, e}$ ). Furthermore, the effectiveness of such policy measures depend on $v_{i, f}$, a parameter describing the dependence of a firm's innovativeness on outside factors. In other words, the model cannot state whether a certain kind of support of private $R \& D$ is successful. However, if such a support is able to increase the innovativeness of firms and innovations are important in the industry under discussion, such support is an adequate measure in the initial phase of the cluster life-cycle.

The support of start-up activity increases the value of term $a_{t}$, especially if $\gamma_{e, s,} \varphi_{s, e}$ and $\varphi_{s, f}$ are large. Again, since we do not model start-up support in detail, the model has some limitations. It might be financial support, organisational support, education of potential entrepreneurs or any other kind of support. However, if there is support that significantly 
increases the start-up activity (large $\left.\varphi_{s, t}\right)$, the emergence of a cluster becomes more likely if start-ups play an important role in the industry (large $\gamma_{e, s}$ and $\left.\varphi_{s, e}\right)$.

Network organisation and cooperation support is an effective measure in the initial stage of the cluster life-cycle if it succeeds to increase the network and cooperation activity (large $v_{x, f}$ ) and if networking and cooperation is important in the industry under discussion (large $Y_{e, x}$ and $\left.v_{x, e}\right)$. As stated above, we are not able to state how networking and cooperation can be supported effectively. However, the model proves that they are adequate tools in the initial stage.

\section{Expansion phase}

In the expansion phase, two different types of dynamics might occur: Dynamic B and Dynamic C (see Section IV.2). Dynamic B stands for a stabilisation of the actual situation. This means that if no cluster has emerged in the industry and region under discussion, so far, no cluster will emerge in the expansion phase. Dynamic C, instead, causes the emergence of a cluster if it is not already present. Hence, policy measures should attempt to make Dynamic $\mathrm{C}$ more likely. This is achieved by small values of $\alpha$ and large values of $b_{t} . \alpha$ depends on the characteristics of the respective industry as well as the stage of the cluster life-cycle and cannot be influenced by policy measures. It becomes large during the expansion phase so that the impact of policy measures is limited. Impacts are rather given at the beginning of the expansion phase, when $\alpha$ is still smaller. The crucial term to be influenced is $b_{t}$.

Above, we found that the support of networking and cooperation as well as the support of private $\mathrm{R} \& \mathrm{D}$ has no impact on term $b_{t}$. This holds only for support that has an impact as modelled here. However, for such kinds of policy measures we find no effects in the expansion phase so that they are rather ineffective in this phase. Other policy measures 
examined in this paper have the potential to influence the dynamics of cluster formation in the expansion phase.

Education has an impact on $b_{t}$, especially if $\gamma_{e, l}, \gamma_{e, s}$ and $\gamma_{s, l}$ are large. This means that policy measures, which lead to a higher education in the region und discussion, have an influence on the emergence of clusters in the expansion phase of the cluster life-cycle. The effectiveness of such measures depends on the industries need for skilled workers (large $Y_{e, l}$ ) as well as on start-up activities and their need for educated potential founders (large $\gamma_{e, s}$ and $\left.\gamma_{s, l}\right)$. Hence, if educated workers are important either for firms or the founding of firms, the support of education is an adequate measure during, and especially at the beginning of the expansion phase.

Similar findings are obtained for public research. Public research is an adequate measure in the expansion phase, too, especially if public research is important for firms in the industry under discussion (large $V_{e, r}$ ) and/or if there is high start-up activity that can be enhanced by public research (large $\gamma_{e, s}$ and $\left.\gamma_{s, l}\right)$.

Moreover, the direct support of start-up activities is effective in the expansion phase of the cluster life-cycle. Again, the effectiveness depends on how much start-up activities can be influenced by policy measures $\left(\varphi_{s, f}\right)$. We are not able to deduce any knowledge about how this can be reached from the model. We find that an increase of the regional start-up activities during the expansion phase also increases the likelihood of the emergence of a cluster.

Infrastructure and local conditions do also matter in the expansion phase of the cluster lifecycle. The effectiveness of policy measures that improve these conditions depends on their relevance for the industrial activity $\left(\gamma_{e, f}\right)$. This means that policy measures have to be adapted to the needs of the industry under consideration in order to be effective. 


\section{Mature phase}

In the mature phase of the cluster life-cycle, the situation changes again. As stated above, in the mature phase, all three kinds of dynamics are possible. Dynamic B is most likely, which implies a stabilisation of the current state. This means that an existing cluster is stabilised, while if no cluster exists, no cluster will emerge. However, the (re-)emergence of a cluster (Dynamic $\mathrm{C}$ ) is possible, if $\alpha$ is small and $b_{t}$ and $c_{t}$ are large. $\alpha$ decreases with time and is not influenced by any policy measures. Hence, the chances that a cluster emerges decrease with time. If $c_{t}$ is small, an existing cluster can run the risk to disappear. Hence, in this phase, policy measures can have two aims. First, they might aim at making the (re-) emergence of a cluster more likely. Second, they might aim at avoiding the disappearance of an existing cluster. In both cases the value of $c_{t}$ is important. Therefore, it is important to recognise that term $c_{t}$ depends on the market condition $\left(m_{t}\right)$. In the mature phase, strong competition and decreasing markets make the success of any policy measure less likely.

Furthermore, there are differences between the two policy aims. Avoiding the disappearance of an existing cluster requires keeping $c_{t}$ sufficiently large, while making the (re-) emergence of a cluster more likely requires making $b_{t}$ and $c_{t}$ large.

Hence, in contrast to the expansion phase of the cluster life-cycle, supporting the (re-) emergence of a cluster in the mature phase requires not only increasing $b_{t}$, but also increasing $c_{t}$. This means that policy has either to choose a measure that increases both terms, $b_{t}$ and $c_{t}$, at the same time or to choose measures that are discussed for the expansion phase and combine them with further measures.

The only measure able to influence $b_{t}$ and $c_{t}$ at the same time is public research. Hence, if innovations are important in the respective industry, if public research matters for innovative activities and if spin-offs from public research play a role, increasing public 
research makes the (re-)emergence of a cluster more likely.

Besides, in the mature phase, the (re-)emergence of a cluster can be made more likely by combining one of the measures from the expansion phase - education, support for startups, and improvement of the infrastructure and local conditions - with support for private $R \& D$, networking or cooperation, which leads to an increase in $c_{t}$. This shows that it is more difficult to trigger the emergence of clusters in the mature phase than in the expansion phase. Furthermore, the potential of such an influence decreases with time. The effectiveness of the different measures depends on the same parameters and thus circumstances for each of the measures as discussed above.

To avoid the disappearance of an existing cluster in the mature phase of the cluster lifecycle, the term $c_{t}$ has to be large. The term $c_{t}$ might become small in the mature phase due to bad market conditions. Policy measures have to balance the negative effects. According to the model, three measures have an impact on $c_{t}$ and, thus, have the potential of a positive impact that balances the negative market developments. These are public research, support for private $R \& D$ and support of networking and cooperation.

Public research is especially effective if innovations are important (large $\gamma_{e, i}$ and $v_{i, e}$ ) and public research contributes significantly to the innovation activities (large $\left.v_{i, r}\right)$. The former also holds for the support of private R\&D, while the effectiveness of outside support of the firms' innovation activities is also important (large $v_{i, r}$ ). If networks and cooperation are important in the industry (large $y_{e, x}$ and $v_{x, e}$ ) and if policy can influence networks and cooperation significantly (large $\left.v_{x, t}\right)$, the support of networking and cooperation is effective.

\section{Summary}

Summing up the results, we first have to state that these results only hold for policy measures that function as considered in the model, e.g. in the model, networking is 
assumed to have an effect on the growth of existing firms. If policy makers support network activities that are especially helpful for founders and, therefore, increase the startup activity, this is not included in the model in the form of network support. It is rather included in the start-up support, which represents all actions that are able to increase the start-up activity.

Furthermore, the theoretical model is not able to tell us whether policy measures do have an effect, at all, and how these measures have to be designed in order to be most effective. The effect of policy measures is represented in the model by parameters. The size of these parameters is an empirical question that is not treated in this paper.

The theoretical results are summarised in Table 3. There is only one measure which seem to be adequate in all stages of the cluster life-cycle: public research. For all other measures we were able to find stages in which they should be applied and stages in which they can be expected to have no effect. We also found that policy measures are, in general, most effective in the initial phase and at the beginning of the expansion phase. They also might help to avoid the decline of an existing cluster. Triggering the emergence of a cluster in the mature phase is possible but most unlikely since it requires severe policy intervention with no guarantee of success.

Besides public research, in the initial phase, the support of start-ups, private R\&D and networking and cooperation should be the measures of choice. This is very well in line with the approaches that are taken in reality as listed in Table 1.

In the expansion phase, our theoretical analysis suggests to provide education, public research, infrastructure and other supportive local conditions as well as to support start-up activities. This differs from the empirical findings. In this stage of development, most cluster policies focus on education, start-up activities and networking (see Table 1). Hence, there is some overlap. However, public research and local conditions are less frequently addressed by policy than the theoretical analysis suggests, while networking is supported, 
although our analysis does not show the effectiveness of this measure.

Talking about networking, we have to be cautious with our interpretation of the model's results. We only modelled the direct effects of networks on the growth of firms. For this kind of networking, we do not find effects in the expansion phase of the cluster life-cycle. Other kinds of networking, for example supporting start-up activities, might well be adequate in this stage.

Table 3: Effects of various policy measures in the different phases of the cluster life-cycle according to the theoretical model

In the mature phase, we find that the emergence of clusters is a rather rare event. Hence, policy measures that try to make the emergence of clusters more likely are rarely successful in this stage. Three measures can prevent existing clusters from disappearance: public research, supporting private R\&D, supporting networking and cooperation. Especially public research and the support of networking are measures frequently applied in reality (Table 1). However, we find that education and improving local conditions are further measures applied in this stage. Our theoretical analysis implies that these measures might make the (re-)emergence of clusters more likely but that this is, in general, a quite rare event.

\section{Conclusion}

This paper examines the effects of policy measures applied in the context of clusters A) with respect to the different stages of the cluster life-cycle and B) their dependence on the cluster's life-cycle stage. We use two approaches. First, the literature on policy measures that are applied in the context of clustering is scanned for the kind of measures that are 
applied in the different stages of the cluster life-cycle. Second, we set up a mathematical model that describes the dynamics of clustering. The parameters are estimated empirically; moreover, the parameters' dependence on the life-cycle stages is identified. Afterwards, the resulting model is used to study the impact of the various policy measures in the different life-cycle stages of clusters.

We find that the literature rarely addresses the question of how policy measures depend on the life-cycle stages explicitly. Nevertheless, the literature implicitly provides some insights about what measure should be used at which stage. In total, we study six different policy categories: education, public research, support of R\&D, support of start-ups, support of networks and cooperation, and other local conditions. The insights from literature distinguish these measures further.

The model's results do partly match the findings from the literature. However, the two approaches have a different perspective. While the mathematical model helps to estimate the strength of the impact of each policy measure with respect to the different stages of the cluster life-cycle, the literature gives information about how measures should be designed in each life-cycle stage. Both information sources seems to be very valuable for policy makers.

Education and the improvement of local conditions show the strongest impact in the expansion phase. In this and other early phases, the literature recommends general training while in more mature phases training for a specialized labour force seems to be more important. Supportive services are usually provided in the mature phase. In the context of the model, this can be seen as improving local conditions. This implies that the current policy is not in line with our theoretical findings. However, as mentioned above, we have to be careful with such an interpretation since the model might not represent the effects of such policy measures correctly.

Public research seems to have a strong impact during the whole cluster life-cycle. 
However, the emergence of a cluster can probably not be triggered in the mature phase of the industrial life-cycle. The literature recommends establishing research institutes especially in the initial and the mature stages, with a stronger focus on the initial phase. This does only partially correspond to the theoretical findings.

In our model examination, the support of private R\&D as well as the support of networks and cooperation is found to have the strongest impact in the initial and mature phases. The literature qualifies this by arguing for different forms of this measure in the various lifecycle phases. In the initial phase, it is recommended to support cooperation and private R\&D. Coordination, the set-up of networks and the support of screening processes for technologies are said to be adequate measures in the expansion phase. In the mature phase, the focus should shift to the renewal of networks.

According to our model analysis, supporting start-up activities is most effective in the early phases of the cluster life-cycle. It also plays a less important role in the mature phase according to the literature. However, the literature distinguishes between promoting and funding start-ups, which is more important in the beginning, and supporting spin-offs, which seems to be more important in the expansion phase.

This paper is the first that combines two perspectives in order to answer the question of which policy measure is most adequate in which phase of the cluster life-cycle. Of course, this paper is only a first step. It can be expected that more policy evaluation will be conducted in the future so that more evidence will become available. The paper's theoretical examination is based on a mathematical model and knowledge about the parameters in this model. Estimates for all parameters for each life-cycle stage would allow for calculating the impacts of policy measure in more detail. Further studies of industries and their development over time would be necessary to obtain such estimates. This is another interesting path for further research. 
Acknowledgements:

We would like to thank Dirk Fornahl as well as other participants of the EMAEE Conference 2009 for helpful comments and suggestions on an earlier draft of this paper. The article has also benefited from detailed comments of two anonymous referees. The usual disclaimers apply. 


\section{References}

AUDRETSCH D. B. and FELDMAN M. P. (1996) Innovative clusters and the industry life cycle, Review of Industrial Organization 11, 253-273.

AUDRETSCH D. B. and LEHMANN E. E. (2006) The role of clusters in knowledge creation and diffusion: an institutional perspective, in ASHEIM B., COOKE P. and MARTIN R. (Eds) Clusters and Regional Development. Critical reflections and explorations, pp. 188-197.Routledge, New York.

ANDERSSON T., SERGER S., SÖRVIK J. and HANSSON E. (2004) The Cluster Policies Whitebook. IKED: Malmö.

ARTHUR W. B. (1994) Increasing Returns and Path Dependence in the Economy. University of Michigan Press, Ann Arbor.

BATHELT H., MALMBERG A., MASKELL P. (2004) Clusters and knowledge: local buzz, global pipelines and the process of knowledge creation, Progress in Human Geography, $28,31-56$.

BATHELT H. and DEWALD U. (2008) Ansatzpunkte einer relationalen Regionalpolitik und Clusterförderung, Zeitschrift für Wirtschaftsgeographie, 53 (2-3), 129-145.

BORRÁS S. and TSAGDIS D. (2008) Cluster Policies in Europe. Firms, Institutions, and Governance. Edward Elgar, Cheltenham, Northampton.

BOSCHMA R. and WENTING R. (2007) The spatial evolution of the British automobile industry: Does location matter?, Industrial and Corporate Change, 16, 213-238.

BRENNER T. (2001) Self-Organisation, Local Symbiosis of Firms and the Life Cycle of Localised Industrial Clusters, Papers on Economics \& Evolution \#0103, Max Planck Institute of Economics, Jena.

BRENNER T. (2006) An Identification of Local Industrial Clusters in Germany, Regional 
Studies, 40, 991-1004.

BRENNER T. (2008) Cluster dynamics and policy implications, Zeitschrift für Wirtschaftsgeographie, 53 (2-3), 146-162.

BRENNER T. and BRÖKEL T. (2009) Methodological Issues in Measuring Innovation Performance of Spatial Units, Working Papers on Innovation and Space \#01.09,

Department of Economic Geography, Philipps-University Marburg, Marburg.

BRENNER T. and FORNAHL D. (2008) Regional Path-Dependence in Start-up Activity, Papers in Evolutionary Economic Geography \#08.12, University of Utrecht, Utrecht.

BRENNER T. and MÜHLIG A. (2007) Factors and Mechanisms Causing the Emergence of Local Industrial Clusters - A Meta-Study of 159 Cases, Papers on Economics \& Evolution \#0723, Max Planck Institute of Economics, Jena.

BRUSCO S. (1990) The idea of the Industrial District: Its genesis, in PYKE F., BECATTINI, G. and SENGENBERGER W. (Eds) Industrial Districts and Inter-firm Co-operation in Italy, pp.10-20. International Institute for Labour Studies, Geneva.

BUSOM I. (1999) An Empirical Evaluation of the Effects of R\&D Subsidies, Burch Center Working Paper No. B99/05, Department of Economics, University of California, Berkeley. CHESHIRE P. (2003) Territorial Competition: Lessons for (Innovation) Policy, in BRÖCKER J., DOHSE D. and SOLTWEDEL R. (Eds) Innovation Clusters and Interregional Competition, pp. 331-347. Springer, Heidelberg, Berlin, New York.

DINNETZ M. (2007) Country Report: Sweden. Oxford Research AS, Frederiksberg.

DTI (2004) A Practical Guide to Cluster Development. A Report to the Department of Trade and Industry and the English RDAs by Ecotec Research \& Consulting. Ecotec, Birmingham. 
ENICHLMAIR C. and OBERHOLZNER T. (2007) Country Report: Austria. Oxford Research AS, Frederiksberg.

ENRIGHT M. (2003) Regional Cluster: What We Know and What We Should Know, in BRÖCKER J., DOHSE D. and SOLTWEDEL R. (Eds) Innovation Clusters and Interregional Competition, pp. 99-129. Springer, Heidelberg, Berlin, New York.

EUROPEAN COMMISSION (2008) The concept of clusters and cluster policies and their role for competiveness and innovation: Main statistical results and lessons learned. Commission Staff Working Document SEC (2008) 2637, Europe INNOVA / PRO INNO Europe paper $\mathrm{N}^{\circ} 9$. European Communities, Luxembourg.

FOUNDATION FOR ENTREPRENEURSHIP DEVELOPMENT (2007) Country Report: Bulgaria. Oxford Research AS, Frederiksberg.

FRITSCH M. and SLAVTCHEV V. (2005) The role of regional knowledge sources for innovation - An empirical assessment. Working Paper 15/2005, Faculty of Economics and Business Administration, Technical University Bergakademie Freiberg, Freiberg.

FROMHOLD-EISEBITH M. and EISEBITH G. (2005) How to institutionalize innovative clusters? Comparing explicit top-down and implicit bottom-up approaches, Research Policy, 34, 1250-1268.

GLAESER E., KALLAL H., SCHEINKMAN J. and SHLEIFER A. (1992) Growth in Cities, Journal of Political Economy, 100 (6), 1126-1152.

HAUSER C. (2007) Country Report: Germany. Oxford Research AS, Frederiksberg.

HENDERSON V., KUNCORO A. and TURNER M. (1995) Industrial Development in Cities, Journal of Political Economy, 103 (5), 1067-1090.

HOSPERS G. and BEUGELSDIJK S. (2002) Regional Cluster Policies: Learning by Comparing?, Kyklos, 55, 381-402.

HUGGINS R. (2008) The Evolution of Knowledge Clusters: Progress and Policy, Economic 
Development Quarterly, 22, 277-289.

JAFFE A. (1999) Measurement Issues, in BRANSCOMB L. and KELLER J. (Eds) Investing in Innovation. Creating a research and innovation policy that works, pp.64-84. MIT Press Cambridge, London.

KIESE M. (2008) Mind the Gap. Regionale Clusterpolitik im Spannungsfeld von Wissenschaft, Politik und Praxis aus der Perspektive der Neuen Politischen Ökonomie, Zeitschrift für Wirtschaftsgeographie, 53 (2-3), 129-145.

KLEPPER S. (1997) Industry Life Cycles, Industrial and Corporate Change, 6, 145-181. KLEPPER S. (2007) The evolution of geographic structures in new industries, in FRENKEN K. (Eds) Applied evolutionary economics and economic geography, pp. 69-92. Edward Elgar, Cheltenham.

KOSCHATZKY K. (2005) The regionalization of innovation policy: new options for regional change?, in FUCHS G. and SHAPIRA P. (Eds) Rethinking Regional Innovation and Change. Path Dependency or Regional Breakthrough?, pp. 291- 312. Springer, New York. LONGHI C. (1999) Networks, Collective Learning and Technology Development in Innovative High Technology Regions: The Case of Sophia-Antipolis, Regional Studies, 33 (4), 333-342.

LUNDEQUIST P. and POWER D. (2002) Putting Porter into Practice? Practices of Regional Cluster Building: Evidence from Sweden, European Planning Studies,10, 685704.

MAGGIONI M. (2004) The Rise and Fall of Industrial Clusters: Technology and the Life Cycle of Regions, Document de treball 2004/6, Universitat de Barcelona, Barcelona. MARTIN R. and SUNLEY P. (2003) Deconstructing clusters: Chaotic concept of policy panacea?, Journal of Economic Geography, 3, 5-35. 
MAS G. (2007) Country Report: United Kingdom. Oxford Research AS, Frederiksberg.

MASKELL P. and KEBIR L. (2006) What qualifies a cluster theory?, in ASHEIM B., COOKE P. and MARTIN R. (Eds) Clusters and Regional Development. Critical reflections and explorations, pp. 30-49. Routledge, New York.

MENZEL M. and FORNAHL D. (2007) Cluster Life Cycles-Dimensions and Rationales of Cluster Development, Jena Economic Research Papers, 2007-076, Friedrich-SchillerUniversity and Max Planck Institute of Economics, Jena.

MÜLLER A. (2007) Country Report: Spain. Oxford Research AS, Frederiksberg.

PAPADOPOULOS C. (2007) Country Report: Cyprus. Oxford Research AS, Frederiksberg.

PORTER M. E. (1990) The competitive advantage of nations. Edward Elgar, New York.

PORTER M. E. (1998a) On Competition. Harvard Business School, Boston.

PORTER M. E. (1998b) Clusters and the new economics of competition, Harvard Business Review, November-December, 77-90.

PORTER M. E. (2003) The Economic Performance of Regions, Regional Studies, 37, 549578.

RAINES P. (2000) Developing Cluster Policies in Seven European Regions, Regional and Industrial Policy Research Paper, 42, European Policies Research Centre, University of Strathclyde, Glasgow.

ROELANDT T. J. A. and DEN HERTOG P. (1999) Cluster Analysis and Cluster-Based Policy Making: The State of The Art, in OECD (Eds) Boosting Innovation: The Cluster Approach, pp. 413-427. OECD, Paris.

SCHLUMP C. and BRENNER T. (2010) University Education, Public Research and Employment Growth in Regions - An Empirical Study of Germany. Working Paper on 
Innovation and Space, \# 02.10, Department of Economic Geography, Philipps-University Marburg, Marburg.

SCHOU S. (2007) Country Report: Denmark. Oxford Research AS, Frederiksberg.

SFORZI F. (1990) The quantitative importance of Marshallian industrial districts in the Italian economy, in PYKE F., BECATTINI G. and SENGENBERGER W. (Eds) Industrial Districts and Inter-firm Co-operation in Italy, pp.75-107. International Institute for Labour Studies, Geneva.

SMALLBONE D., NORTH D. and VICKERS I. (2003) The role and characteristics of SMEs in innovation, in ASHEIM B., ISAKSEN A., NAUWELAERS C. and TÖDTLING F. (Eds) Regional Innovation Policy For Small-Medium Enterprises, pp. 3-20. Edward Elgar, Cheltenham, Northampton.

SÖLVELL Ö., LINDQUIST G., KETELS C. (2003) The Cluster Initiatives Greenbook. Ivory Tower, Stockholm.

STERNBERG R. (2003) New Firms, Regional Development and the Cluster Approach What Can Technology Policies Achieve?, in BRÖCKER J., DOHSE D. and SOLTWEDEL R. (Eds) Innovation Clusters and Interregional Competition, pp. 347-372. Springer, Heidelberg, Berlin, New York.

STERNBERG R. and LITZENBERGER T. (2004) Regional Clusters in Germany: Their Geography and their Relevance for Entrepreneurial Activities, European Planning Studies, 12, 767-792.

VAN KLINK A. and DE LANGEN P. (2001) Cycles in industrial clusters: The case of the shipbuilding industry in the Northern Netherlands, Tijdschrift Voor Economische En Sociale Geografie, 92, 449-463.

VAN DER LINDE C. (2003) The Demography of Clusters - Findings from the Cluster Meta-Study, in BRÖCKER J., DOHSE D. and SOLTWEDEL R. (Eds) Innovation Clusters 
and Interregional Competition, pp.130-149. Springer, Heidelberg, Berlin, New York.

WOLFE D. A. and GERTLER M. S. (2006) Local Antecedents and Trigger Events: Policy Implications of Path Dependence for Cluster Formation, in BRAUNERHJELM P. and FELDMAN M. (Eds) Cluster Genesis. Technology-Based Industrial Development, pp. 243263. University Press, Oxford. 
Appendix

List of parameters:

Table A.1: List of the parameters with their median estimation (for seven empirically examined industries in Germany), the theoretical prediction of their relative strength (according to Section II.1) and information whether the theoretical prediction is confirmed by the empirical study. If the empirical estimation was not possible or a parameter does not change during the cluster life-cycle, "-" is used

Equations:

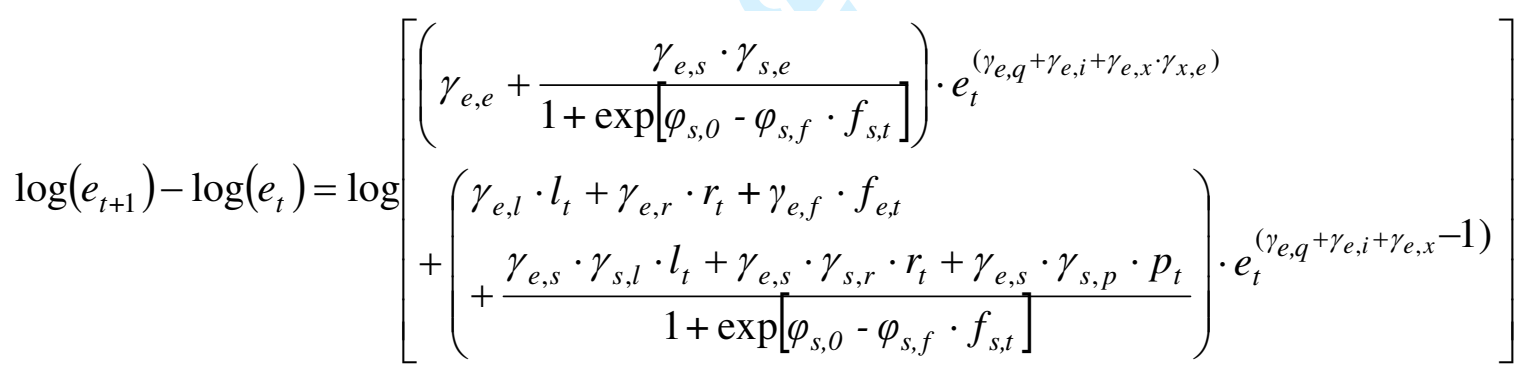

$$
\begin{aligned}
& +\log \left[\left(\frac{p_{t}-e_{t}}{p_{t}}\right)^{\gamma_{e, p}}\right]+\log \left[\begin{array}{l}
\left(\frac{E_{g l o b, t}}{e_{g l o b, t} \cdot E_{t}}\right)^{\gamma_{e, q}} \cdot m_{t}^{\gamma_{e, m}} \cdot\left(\frac{v_{i, e}}{1+\exp \left[v_{i, 0}-\left(v_{i, r} \cdot r_{t}+v_{i, f} \cdot f_{i, t}\right)\right]}\right) \\
\left.\cdot\left(\frac{v_{x, e}}{1+\exp \left[v_{x, 0}-v_{x, f} \cdot f_{x, t}\right]}\right)^{\gamma_{e, x}}\right]
\end{array}\right]
\end{aligned}
$$

$$
\alpha=\gamma_{e, q}+\gamma_{e, i}+\gamma_{e, x} \cdot \gamma_{x, e}
$$




$$
\begin{gathered}
a_{t}=\gamma_{e, e}+\frac{\gamma_{e, s} \cdot \varphi_{s, e}}{1+\exp \left[\varphi_{s, 0}-\varphi_{s, f} \cdot f_{s, t}\right]} \\
b_{t}=\gamma_{e, l} \cdot l_{t}+\gamma_{e, r} \cdot r_{t}+\gamma_{e, f} \cdot f_{e, t}+\frac{\gamma_{e, s} \cdot \gamma_{s, l} \cdot l_{t}+\gamma_{e, s} \cdot \gamma_{s, r} \cdot r_{t}+\gamma_{e, s} \cdot \gamma_{s, p} \cdot p_{t}}{1+\exp \left[\varphi_{s, 0}-\varphi_{s, f} \cdot f_{s, t}\right]} \\
c_{t}=\left[\begin{array}{l}
\left.\left(\frac{E_{g l o b, t}}{e_{g l o b, t} \cdot E_{t}}\right)^{\gamma_{e, q}} \cdot m_{t}^{\gamma_{e, m}} \cdot\left(\frac{v_{i, e}}{1+\exp \left[v_{i, 0}-\left(v_{i, r} \cdot r_{t}+v_{i, f} \cdot f_{i, t}\right)\right]}\right)^{\gamma_{e, i}}\right] \\
\left.\left(\frac{v_{x, e}}{1+\exp \left[v_{x, 0}-v_{x, f} \cdot f_{x, t}\right]}\right)^{\gamma_{e, x}}\right]
\end{array}\right.
\end{gathered}
$$

Median values of abbreviations:

Table A.2: Dependence of terms $\alpha, a_{t}, b_{t}$ and $c_{t}$ on the stages of the cluster life-cycle

Proof of Proposition 1:

In order to identify the stable states of the dynamics given by Equation (10), we first have to determine the stationary states, meaning the states with $d e_{t} / d t=0$. These states are given by

$$
\log \left[c_{t} \cdot\left(a_{t} \cdot e_{t}^{\alpha}+b_{t} \cdot e_{t}^{\alpha-1}\right) \cdot\left(\frac{p_{t}-e_{t}}{p_{t}}\right)^{\gamma_{e, p}}\right]=0
$$

Let us first consider $\alpha<1$. For $e_{t} \longrightarrow 0$ the term above (left-hand side of (A.6)) goes to infinity. For $e_{t} \rightarrow p_{t}$ the term above goes to $-\infty$. Furthermore, the term is continuous in $e_{t}$. Hence, there is at least one value of $e_{t}$ for which the term takes a value of 0 . If the term has local minima and maxima, there might be more values of $e_{t}$ for which the term on the left-hand side of (A.6) equals 0. In order to find the local minima and maxima of the left-hand side of 
(A.6) we consider the term $L\left(e_{t}\right)$ which is given by Equation (11):

$$
L\left(e_{t}\right)=c_{t} \cdot\left(a_{t} \cdot e_{t}^{\alpha}+b_{t} \cdot e_{t}^{\alpha-1}\right) \cdot\left(\frac{p_{t}-e_{t}}{p_{t}}\right)^{\gamma_{e, p}} .
$$

We look for values of $e_{t}$ for which the derivative of this term is zero. For small values of $e_{t}$, according to Assumption 1, the last term of $L\left(e_{t}\right)$ can be ignored. Hence, a local minima or maxima for small values exists if

$$
c_{t} \cdot\left(a_{t} \cdot \alpha \cdot e_{t}^{\alpha-1}+b_{t} \cdot(\alpha-1) \cdot e_{t}^{\alpha-2}\right)=0
$$

This condition is satisfied for

$$
\hat{e}_{1}=\frac{b_{t} \cdot(1-\alpha)}{a_{t} \cdot \alpha}
$$

For large values of $e_{t}$, according to Assumption 1, the first term in $L\left(e_{t}\right)$ can be ignored. Hence, a local minima or maxima for large values exists if

$$
c_{t} \cdot b_{t} \cdot e_{t}^{\alpha-1} \cdot\left(\frac{p_{t}-e_{t}}{p_{t}}\right)^{\gamma_{e, p}} \cdot\left(\frac{\alpha-1}{e_{t}}-\frac{\gamma_{e, p}}{p_{t}-e_{t}}\right)=0 .
$$

This condition is satisfied for

$$
\hat{e}_{2}=\frac{\alpha-1}{\gamma_{e, p}+\alpha-1} p_{t}
$$

Hence, the term $L\left(e_{t}\right)$ has one local minimum at a value of $\hat{e}_{1}$ and one local maximum at a value of $\hat{e}_{2}$. Three situations might result:

1. $L\left(\hat{e}_{2}\right)<1$, which implies also $L\left(\hat{e}_{1}\right)<1$ : This implies that Equation (A.6) cannot be satisfied for value of $e_{t}$ larger than $\hat{e}_{1}$. Hence, there is only one stationary state, which is stable for a low value of $e_{t}\left(<\hat{e}_{1}\right)$.

2. $L\left(\hat{e}_{1}\right)<1$ and $L\left(\hat{e}_{2}\right)>1$ : This implies that Equation (A.6) is satisfied for values of $e_{t}$ between $\hat{e}_{1}$ and $\hat{e}_{2}$ as well as for values of $e_{t}$ smaller than $\hat{e}_{1}$ and values of $e_{t}$ larger than $\hat{e}_{2}$. Hence, there are three stationary states. The lowest $\left(e_{t}<\hat{e}_{1}\right)$ and the highest $\left(e_{t}>\hat{e}_{2}\right)$ of them are stable. 
3. $L\left(\hat{e}_{1}\right)>1$, which implies also $L\left(\hat{e}_{2}\right)>1$ : This implies that Equation (A.6) cannot be satisfied for values of $e_{t}$ smaller than $\hat{e}_{2}$. Hence, there is only one stationary state, which is stable, for a high value of $e_{t}\left(>\hat{e}_{2}\right)$.

The situation is different if $\alpha>1$. In this case the term on the left-hand side of (A.6) is $-\infty$ for $e_{t} \rightarrow 0$ and for $e_{t} \rightarrow p_{t}$. This implies that one stable stationary state exists for $\hat{e}_{1}=0 . L\left(e_{t}\right)$ has no local minima but has a local maximum at $\hat{e}_{2}$ given by (A.11). Two different situations result: First, if $L\left(\hat{e}_{2}\right)<1$, there is only one stable state for $e_{t}=0$ as described as situation 1 above. Second, if $L\left(\hat{e}_{2}\right)>1$, there are three stationary states as described as situation 2 above. 


\begin{tabular}{|c|c|}
\hline $\begin{array}{l}\text { Most relevant phase in the cluster } \\
\text { life-cycle }\end{array}$ & Policy measure \\
\hline Initial & $\begin{array}{l}\text { Start-up promotion (science parks, incubators) } \\
\text { Cooperation support (meetings etc.) } \\
\text { Development of innovative culture } \\
\text { Establishment of laboratories } \\
\text { R\&D support }\end{array}$ \\
\hline Initial/Expansion & $\begin{array}{l}\text { Education and training (conferences, learning } \\
\text { processes) } \\
\text { Networks (informal, institutionalised), joint activities } \\
\text { Seed funds, better access to capital, VC } \\
\text { Cluster marketing \& service provision }\end{array}$ \\
\hline Expansion & $\begin{array}{l}\text { Screening activities, access to new technology } \\
\text { Industrial coordination and cooperation }\end{array}$ \\
\hline Expansion/Mature & Spin-off support (financing, collaborations, services) \\
\hline Mature & $\begin{array}{l}\text { Development of human capital, specialized work } \\
\text { force } \\
\text { Support services } \\
\text { Renewal of networks, cooperations } \\
\text { Lighthouse projects } \\
\text { Set-up of research institutes }\end{array}$ \\
\hline
\end{tabular}


Table 1: Policy measures and the time in the cluster life-cycle when they are most relevant 


\begin{tabular}{|c|c|c|}
\hline Stage & Dynamics & Conditions \\
\hline \multirow[t]{2}{*}{ Initial stage } & Emergence of a cluster $(\mathrm{C})$ & $a_{t}$ and $c_{t}$ rather large \\
\hline & No emergence of a cluster (A) & $a_{t}$ and $c_{t}$ rather small \\
\hline \multirow[t]{2}{*}{$\begin{array}{l}\text { Expansion } \\
\text { stage }\end{array}$} & Emergence of a cluster $(\mathrm{C})$ & $\begin{array}{l}\alpha \text { rather small, } b_{t} \text { rather } \\
\text { large }\end{array}$ \\
\hline & Stabilisation of the situation (B) & $\begin{array}{l}\alpha \text { rather large, } b_{t} \text { rather } \\
\text { small }\end{array}$ \\
\hline \multirow[t]{3}{*}{ Mature stage } & (Re-)emergence of a cluster (C) & $\begin{array}{l}\alpha \text { small and } b_{t} \text { and } c_{t} \\
\text { rather large }\end{array}$ \\
\hline & Stabilisation of the situation (B) & $\begin{array}{l}\alpha \text { and } c_{t} \text { rather large } \\
\text { and } b_{t} \text { rather small }\end{array}$ \\
\hline & Disappearance of a cluster (A) & $c_{t}$ not large \\
\hline
\end{tabular}

Table 2: Model implications for the dynamics in the three stages of the cluster life-cycle 


\begin{tabular}{|l|c|c|c|c|}
\hline \multirow{2}{*}{ Policy measure } & \multicolumn{3}{|c|}{ Impact in } \\
\cline { 2 - 5 } & Initial phase & Expansion & \multicolumn{2}{|c|}{ Mature phase for } \\
\cline { 3 - 5 } & & phase & Avoiding decline & Triggering \\
energence
\end{tabular}

Table 3: Effects of the various policy measures in the different phases of the cluster lifecycle according to the theoretical model 


\begin{tabular}{|c|c|c|c|c|c|}
\hline \multirow{2}{*}{ Parameter } & \multirow{2}{*}{ Median } & \multicolumn{3}{|c|}{$\begin{array}{l}\text { Strength according to the theoretical knowledge } \\
\text { (Section II.1) in the different stages }\end{array}$} & \multirow{2}{*}{$\begin{array}{c}\text { Confirmed by } \\
\text { empirical } \\
\text { estimations }\end{array}$} \\
\hline & & Initial stage & $\begin{array}{l}\text { Expansion } \\
\text { stage }\end{array}$ & Mature stage & \\
\hline$\gamma_{e, e}$ & 0.4 & small & very large & medium & No evidence \\
\hline$Y_{e, s}$ & 112 & Very large & large & small & No evidence \\
\hline$Y_{e, l}$ & 0.96 & large & very large & medium & No evidence \\
\hline$V_{e, r}$ & 7.9 & large & very large & medium & yes \\
\hline$Y_{e, f}$ & 200 & large & medium & medium & No evidence \\
\hline$Y_{e, q}$ & 0.5 & small & large & large & yes \\
\hline$V_{e, m}$ & - & - & - & - & - \\
\hline$V_{e, i}$ & 0.001 & medium & very large & medium & No evidence \\
\hline$V_{e, x}$ & - & small & very large & very large & - \\
\hline$Y_{e, p}$ & 2.9 & - & - & - & No evidence \\
\hline$\varphi_{s, p}$ & 0.02 & very large & large & small & yes \\
\hline$\varphi_{s, e}$ & 1.14 & very large & large & small & yes \\
\hline$\varphi_{s, l}$ & 5.4 & very large & large & small & No evidence \\
\hline$\varphi_{s, r}$ & 4.2 & very large & large & small & yes \\
\hline
\end{tabular}




\begin{tabular}{|c|c|c|c|c|c|}
\hline$\varphi_{s, 0}$ & 7.3 & - & - & - & No evidence \\
\hline$\varphi_{s, f}$ & 0.1 & very large & large & small & No evidence \\
\hline$v_{i, e}$ & 55 & small & very large & medium & yes \\
\hline$v_{i, r}$ & 0 & medium & very large & medium & No evidence \\
\hline$v_{i, x}$ & - & small & very large & very large & - \\
\hline$v_{i, f}$ & 0.02 & medium & very large & medium & No evidence \\
\hline$v_{i, 0}$ & 8.8 & - & - & - & No evidence \\
\hline$v_{x, e}$ & - & small & very large & very large & - \\
\hline$V_{x, e}$ & - & - & - & - & - \\
\hline$v_{x, f}$ & - & small & very large & very large & - \\
\hline$m_{t}^{\gamma_{e, m}}$ & - & medium & very large & medium & - \\
\hline
\end{tabular}

Table A.1: List of the parameters with their median estimation (for seven empirically examined industries in Germany), the theoretical prediction of their relative strength (according to Section II.1) and the information whether the theoretical prediction is confirmed by the empirical study. If the empirical estimation was not possible or a parameter does not change during the cluster life-cycle, "-" is used. 


\begin{tabular}{|c|c|c|c|c|}
\hline \multirow[t]{2}{*}{ Abbreviations } & \multirow[t]{2}{*}{ Median } & \multicolumn{3}{|c|}{$\begin{array}{l}\text { Value according to the theoretical knowledge (Section II.1) } \\
\qquad \text { in the different stages }\end{array}$} \\
\hline & & Initial stage & Expansion stage & Mature stage \\
\hline$\alpha$ & 0.5 & small & very large & large \\
\hline$a_{t}$ & 0.52 & $\begin{array}{l}\text { very large, but } \\
\text { the first term } \\
\text { small }\end{array}$ & large - very large & small \\
\hline$b_{t}$ & $\begin{array}{l}1.5^{\star} I_{t}+8.3^{*} r_{t} \\
+0.002^{*} p_{t}+ \\
200\end{array}$ & large - very large & $\begin{array}{l}\text { large - very large, } \\
\text { but the last term } \\
\text { medium }\end{array}$ & small - medium \\
\hline$c_{t}$ & $0.01 \cdot m_{t}^{\gamma_{e, m}}$ & small - medium & very large & $\begin{array}{c}\text { medium, with one } \\
\text { part very large }\end{array}$ \\
\hline
\end{tabular}

Table A.2: Dependence of the terms $\alpha, a_{t}, b_{t}$ and $c_{t}$ on the stages of the cluster life-cycle 


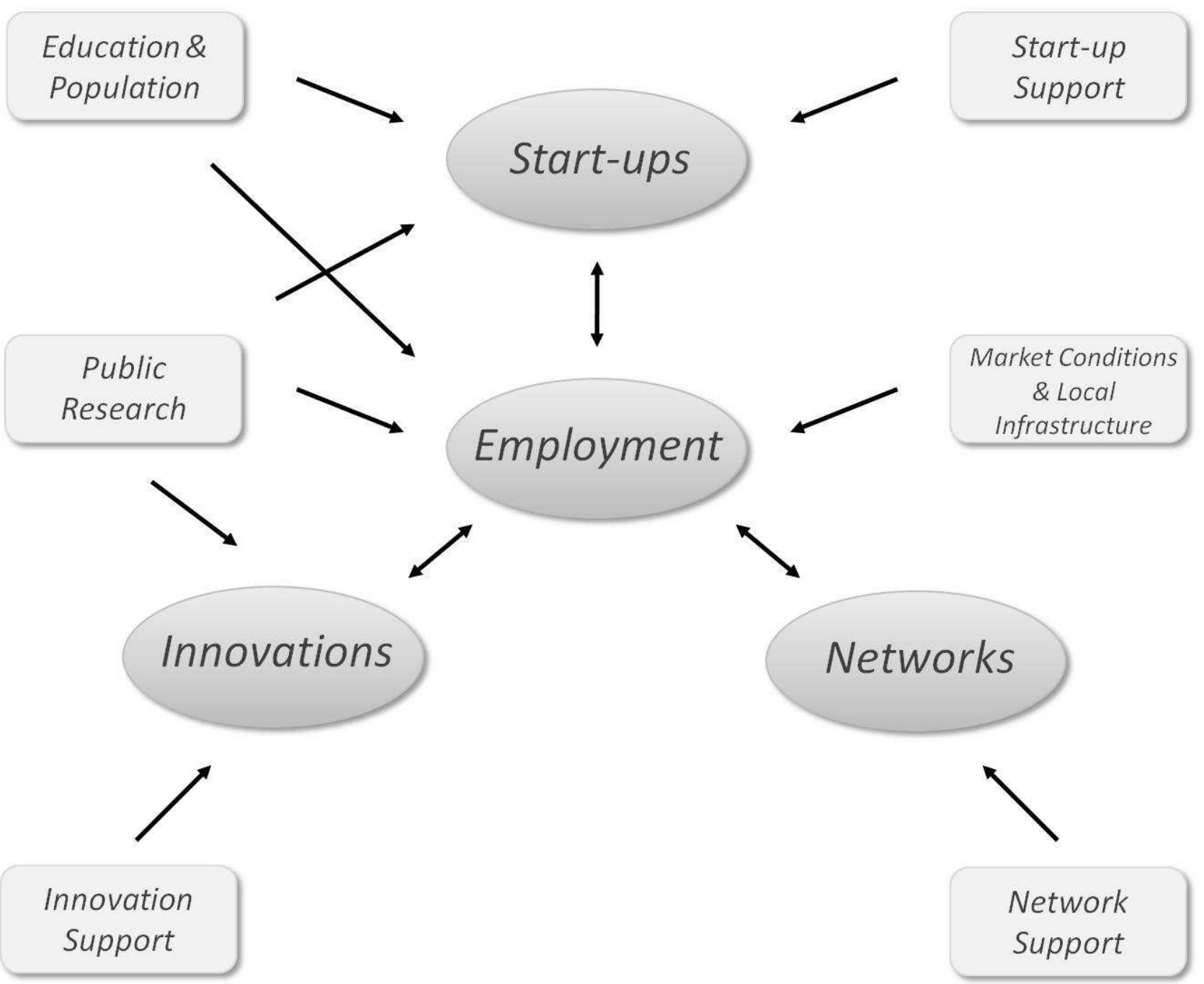

Figure 1: Structure of the mathematical model 
$L\left(e_{t}\right)$

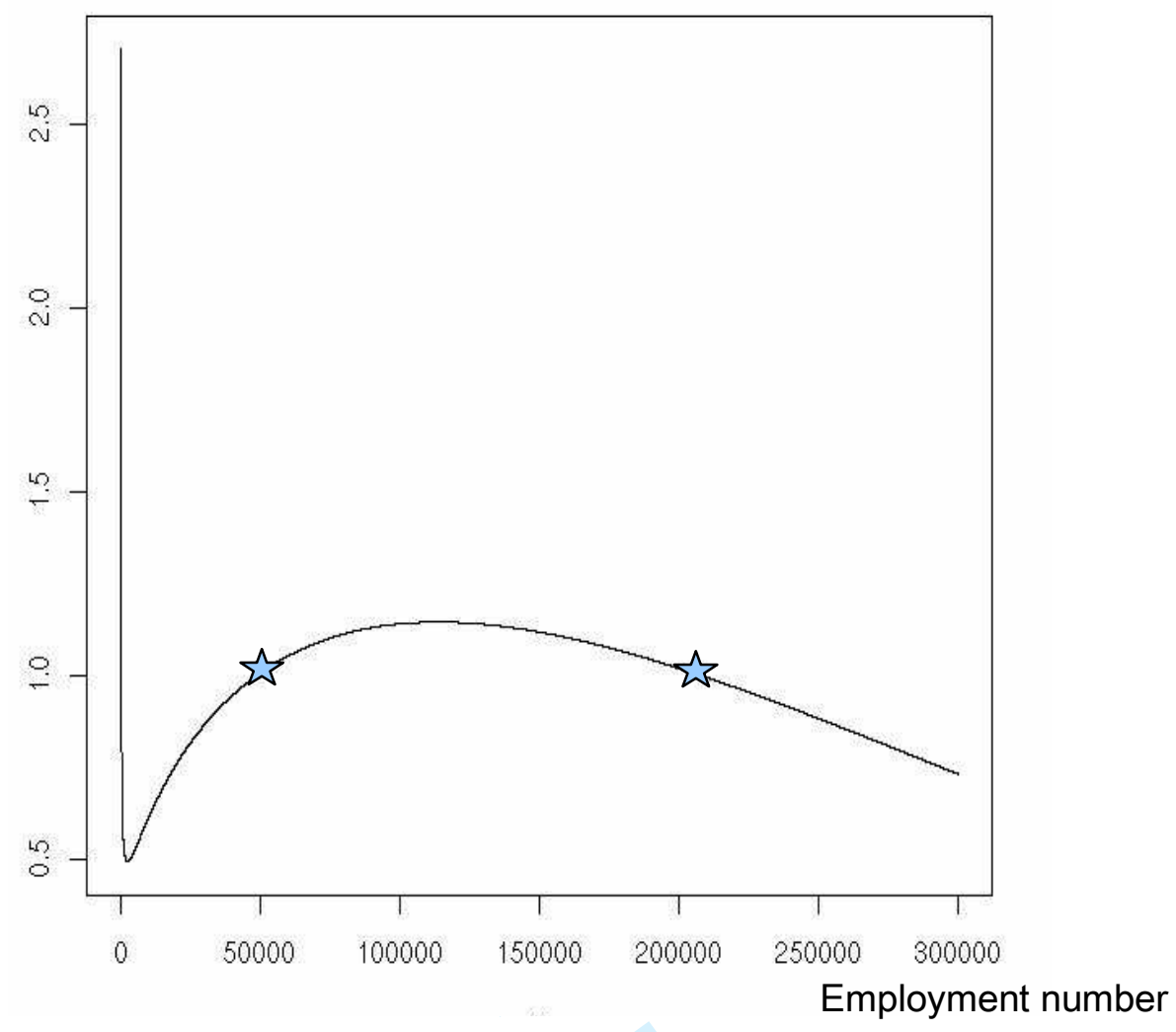

Figure 2: Function $L\left(e_{t}\right)$ for the median parameter values and a region with 800,000 inhabitants. 